\title{
Appetite Control across the Lifecourse: The Acute Impact of Breakfast Drink Quantity and Protein Content. The Full4Health Project
}

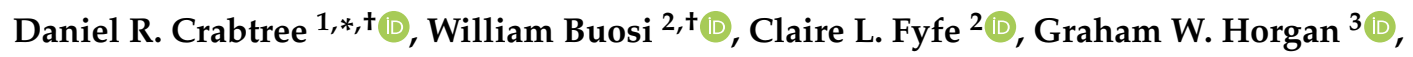 \\ Yannis Manios $\left.{ }^{4}{ }^{(}\right)$, Odysseas Androutsos ${ }^{5}$, Angeliki Giannopoulou ${ }^{4}$, Graham Finlayson ${ }^{6}(\mathbb{D}$, \\ Kristine Beaulieu ${ }^{6}{ }^{(0}$, Claire L. Meek ${ }^{7}$, Jens J. Holst ${ }^{8}{ }^{\circ}$, Klaske Van Norren ${ }^{9}{ }^{\circ}$, \\ Julian G. Mercer ${ }^{2}$, Alexandra M. Johnstone ${ }^{2}$ and on behalf of the Full4Health-Study Group \\ 1 Centre for Health Science, Division of Biomedical Sciences, University of the Highlands and Islands, \\ Old Perth Road, Inverness IV2 3JH, UK \\ 2 The Rowett Institute, University of Aberdeen, Foresterhill Road, Aberdeen AB25 2ZD, UK; \\ william.buosi.m@gmail.com (W.B.); c.fyfe@abdn.ac.uk (C.L.F.); j.mercer@abdn.ac.uk (J.G.M.); \\ alex.johnstone@abdn.ac.uk (A.M.J.) \\ 3 Biomathematics and Statistics Scotland, Foresterhill Road, Aberdeen AB25 2ZD, UK; g.horgan@abdn.ac.uk \\ 4 Department of Nutrition-Dietetics, School of Health Science \& Education, Harokopio University Athens, \\ 70 El. Venizelou Avenue, 17671 Kallithea, Greece; manios@hua.gr (Y.M.); agiann@hua.gr (A.G.) \\ 5 Department of Nutrition and Dietetics, School of Physical Education, Sport Science and Dietetics, \\ University of Thessaly, 42100 Trikala, Greece; oandroutsos@uth.gr \\ 6 School of Psychology, University of Leeds, Leeds LS2 9JT, UK; g.s.finlayson@leeds.ac.uk (G.F.); \\ k.beaulieu@leeds.ac.uk (K.B.) \\ 7 Institute of Metabolic Science, Metabolic Research Laboratories, University of Cambridge, \\ Addenbrooke's Hospital, Box 289, Hills Road, Cambridge CB2 0QQ, UK; clm70@cam.ac.uk \\ 8 Department of Biomedical Sciences and Novo Nordisk Foundation Center for Basic Metabolic Research, \\ University of Copenhagen, DK-2200 Copenhagen, Denmark; jjholst@sund.ku.dk \\ 9 Nutritional Biology, Human Nutrition and Health, Wageningen University, \\ 6708 WE Wageningen, The Netherlands; klaske.vannorren@wur.nl \\ * Correspondence: daniel.crabtree@uhi.ac.uk; Tel.: +44-(0)1-4632-79405 \\ $\dagger$ These authors contributed equally to this work.
}

Received: 16 October 2020; Accepted: 27 November 2020; Published: 30 November 2020

check for updates Abstract: Understanding the mechanisms of hunger, satiety and how nutrients affect appetite
control is important for successful weight management across the lifecourse. The primary aim
of this study was to describe acute appetite control across the lifecourse, comparing age groups
(children, adolescents, adults, elderly), weight categories, genders and European sites (Scotland and
Greece). Participants ( $n=391$ ) consumed four test drinks, varying in composition ( $15 \%$ (normal
protein, NP) and $30 \%$ (high protein, HP) of energy from protein) and quantity (based on $100 \%$
basal metabolic rate (BMR) and $140 \%$ BMR), on four separate days in a double-blind randomized
controlled study. Ad libitum energy intake (EI), subjective appetite and biomarkers of appetite and
metabolism (adults and elderly only) were measured. The adults' appetite was significantly greater
than that of the elderly across all drink types ( $p<0.004)$ and in response to drink quantities ( $p<0.001$ ).
There were no significant differences in EI between age groups, weight categories, genders or sites.
Concentrations of glucagon-like peptide 1 (GLP-1) and peptide YY (PYY) were significantly greater in
the elderly than the adults ( $p<0.001)$. Ghrelin and fasting leptin concentrations differed significantly
between weight categories, genders and sites $(p<0.05)$, while GLP-1 and PYY concentrations differed
significantly between genders only ( $p<0.05)$. Compared to NP drinks, HP drinks significantly
increased postprandial GLP- 1 and PYY $(p<0.001)$. Advanced age was concomitant with reduced
appetite and elevated anorectic hormone release, which may contribute to the development of 
malnutrition. In addition, appetite hormone concentrations differed between weight categories, genders and geographical locations.

Keywords: appetite; lifecourse; gut hormones; hunger; protein

\section{Introduction}

Nutrition-related noncommunicable diseases are associated with increased morbidity and mortality at all stages of life [1,2]. Physiological and psychological responses to food change as we age, with impact on food choices and preferences, but little is known about how appetite control varies across the lifecourse [3]. This is a critical issue in combatting food intake-related chronic disease, commonly driven by over-consumption, but also in consideration of relative under-nutrition in the elderly and the clinically compromised.

Food intake and appetite are governed across the lifecourse by complex interactions between peripherally synthesized gut hormones and their central receptors [4]. These interactions are subject to external influences, including hedonic cues and the environment [5]. Short-acting gastrointestinal signals include the anorexigenic peptides glucagon-like peptide 1 (GLP-1) and peptide YY (PYY) and the orexigenic hormone ghrelin, while leptin maintains long-term energy homeostasis [6]. Homeostatic systems can, however, be overridden by hedonic signals, resulting in appetite control dysfunction, excess energy consumption and obesity [7]. There may be key periods in the lifecourse when appetite can be modulated for optimal health. For example, the onset of overweight and obesity starts as early as childhood and can track into adulthood [8]. Environmental factors contribute towards weight gain, if rewarding energy-dense foods are freely available and integrated into local culture, creating an obesogenic environment [9]. With advancing age, food reward signals are altered [10,11], food craving behavior declines, particularly in females [12] and food intake is suppressed [13,14], all contributing to a condition termed the "anorexia of ageing" [15]. Cross-sectional research reports a peak in calorie intake during late adolescence, followed by a decline, with calorie intake reducing by $1300 \mathrm{kcal} / \mathrm{day}$ on average between 20 and 80 years of age for males and $600 \mathrm{kcal} /$ day for females [16]. Understanding how dietary interventions influence physiological and behavioral mediators of appetite at different stages of life is vital for effective long-term weight control [17]. High-protein diets are often recommended for weight management, as they are highly satiating [18-20], and in the prevention and treatment of malnutrition, particularly in elderly populations [21]. Protein-induced satiety has been observed acutely, within single meals that contained $25 \%$ to $81 \%$ of energy from protein, associated with reductions in subsequent energy intake (EI) compared to lower protein alternatives [22]. In children and adolescents, studies have reported either an appetite suppressant effect of increased protein content [23] or no effect [24]. In addition, breakfasts high in protein have been shown to induce greater hunger suppression compared to breakfasts with a lower protein content in adults [25]. It is not well understood how interactions between protein and appetite control differ between children, adolescents, adults and the elderly since studies are rarely conducted across the lifecourse.

The primary aim of this study was to describe the acute regulation of appetite across the lifecourse, thus being able to detect differences between four different age groups (children, adolescents, adults and elderly), two different weight categories (normal weight and overweight), the two genders (male and female) and two European sites (Aberdeen, Scotland and Athens, Greece). The secondary aim was to examine the short-term effects of breakfast test drinks varying in protein composition and quantity on appetite control. Our study is unique in that it applies an individualized appetite challenge across the lifecourse in lean and overweight males and females in northern and southern Europe. 


\section{Materials and Methods}

\subsection{Participants}

Normal weight and overweight/obese and male and female child, adolescent, adult and elderly participants (age range 7-77 years) were recruited in Scotland and Greece as part of an identical, dual-site within-day dietary intervention study, thereby creating four groups: age (children, adolescents, adults and elderly), weight category (normal weight and overweight), gender (male and female) and site (Scotland and Greece). Recruitment of volunteers was by public advertisement using radio, newspapers and social media, and was conducted from May 2012 to August 2015. When requested, study information sessions were conducted at schools, health care centers and day care centers for the elderly. Participants were individuals who were motivated to actively respond to the volunteer request. Exclusion criteria included: smokers; morbid obesity (BMI $\geq 40 \mathrm{~kg} / \mathrm{m}^{2}$ ); pregnancy; obesity of known endocrine origin; neurological disorders; medication known to influence appetite (including orlistat, oral antidiabetics, insulin, digoxin, anti-arrhythmics, sibutramine, antidepressants); self-reported fever/systemic infection; participation in medical or surgical weight loss program within 1 month of selection; history of cerebrovascular disease; current major depressive disorder; history of cardiovascular disease; chronic obstructive pulmonary disease; an allergy to any of the test drink components and partaking in $>6 \mathrm{~h}$ of vigorous physical activity per week. This study was conducted according to the guidelines laid down in the Declaration of Helsinki [26]. Ethical approval in Aberdeen was granted by the National Health Service North of Scotland Research Ethics Service. Ethical approval in Athens was granted by the Bioethics Committee of Harokopio University and the Greek Ministry of Education for the implementation of the study in schools. The study received ethical approval from NHS Grampian, Aberdeen, Scotland, UK and the Research Ethics Committee (reference number: 12/NS/0007). All participants provided written informed consent before entering the study and, in addition, the parents/guardians of the children consented for their child to participate.

\subsection{Experimental Procedures and Protocol}

Data on children and adolescents were collected at schools and adults and elderly attended the Rowett Institute, University of Aberdeen, Scotland (ABDN) and the Department of Nutrition-Dietetics, Harokopio University Athens, Greece (HUA). Prior to the main experimental trials, preliminary anthropometric measures were carried out under standardized conditions. During the main experimental trials, participants consumed four test drinks for breakfast on four separate occasions using a double-blind randomized controlled crossover design, with at least a 4 day period between trials. On the morning of each trial, participants arrived following an overnight fast (10 h) and having refrained from alcohol consumption and strenuous exercise for $12 \mathrm{~h}$. Test drinks were consumed immediately following baseline measures $(0 \mathrm{~min})$, then at $120 \mathrm{~min}$ post-baseline, ad libitum EI was measured by means of a 30 min buffet-style test meal, after which participants were free to leave. Subjective appetite sensations were assessed using visual analog scales (VASs) at 0, 30, 60, 90 and 120 min (pre-meal), pleasantness and satisfaction VASs were also completed immediately post-test drink. Blood samples were taken at 0,30,60 and 120 min to determine biomarker concentrations. The true aims of the study were concealed from the participants; however, all participants were fully debriefed following their completion of the study. See Figure 1 for an overview of the experimental protocol. This trial was registered at clinicaltrials.gov as NCT01597024. 
$\mathbf{A}$

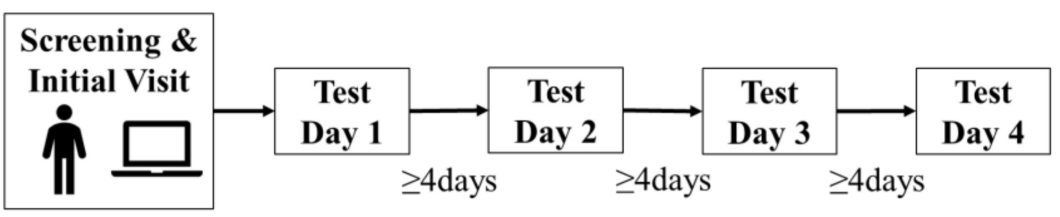

B

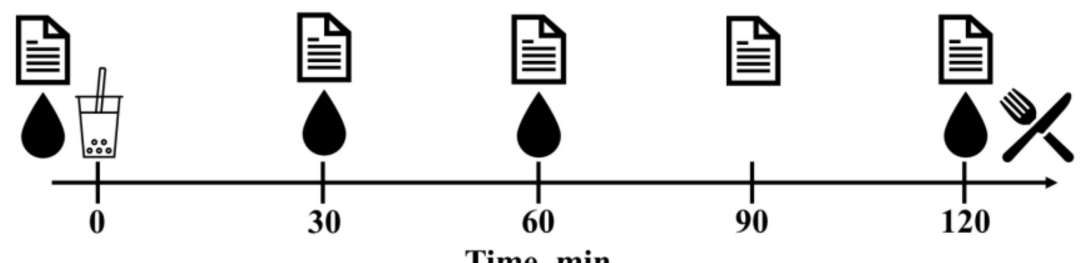

Time, $\min$

Figure 1. Experimental design (A) and test day protocol (B). II anthropometric measurements, $\square$ LFPQ, 目 appetite ratings (Likert scale, children and adolescents; VAS, adult and elderly), sampling (adult and elderly cohorts only), 2 randomized test drink intake (NPMT, NPWL, HPMT or HPWL), 1 ad libitum food intake from buffet-style test meal; (HPMT) high-protein maintenance, HPWL: high-protein weight loss, LFPQ: Leeds Food Preference Questionnaire, NPMT: normal-protein maintenance, NPWL: normal-protein weight loss, VAS: visual analog scale.

\subsection{Anthropometric Measures}

Height, body mass, waist circumference and body composition were measured in the fasted state and after voiding as described previously [27]. Height was measured to the nearest $0.1 \mathrm{~cm}$ using a portable stadiometer (Model 213, SECA, Hamburg, Germany). Body mass, measured to the nearest $0.1 \mathrm{~kg}$, and body composition were assessed using a multi frequency segmental body composition analyzer (Model BC-418-MA, Tanita Corporation, Tokyo, Japan). Body mass index was calculated for each participant and compared against the age- and gender-matched thresholds for normal weight and overweight, as defined by the World Health Organization [28]. In addition, waist circumference and visceral fat percentage measures were performed using abdominal bioelectrical impedance analysis (AB 140 Viscan, Tanita Corporation, Tokyo, Japan), with participants in the supine position.

\subsection{Test Drinks}

The test drinks provided for the study were designed by Nutricia (Danone, Utrecht, The Netherlands) to taste, look and smell identical. One test drink was created with a normal-protein (NP) composition ( $15 \%$ energy from protein) and the other was created with a high-protein (HP) composition ( $30 \%$ energy from protein). The test drink compositions are presented in Table 1 and compared with whole milk. The test drink quantity either corresponded to $100 \%$ of the participant's estimated basal metabolic rate (BMR, kcal/day; weight loss requirements, WL) or 140\% of the participant's estimated BMR (weight maintenance requirements, MT). Basal metabolic rate was estimated for all age groups according to equations derived by Schofield [29], suitable for children and adults (see Table S1 for the equations used to estimate BMR). When calculating participant energy requirements for the MT drinks, BMR was multiplied by a correction factor of 1.4 , whereas when calculating energy requirements for the WL drinks, BMR was multiplied by a correction factor of 1 . In addition, for the purposes of this study, breakfast was defined as the first meal of the day consisting of $25 \%$ of the participant's daily energy requirements, which is similar to previous studies [30,31]. Therefore, when calculating 
participant energy requirements for both drinks, daily BMR was multiplied by 0.25 . The following formulas give the energy requirements (ER) for the MT and WL test drinks, respectively:

$$
\begin{gathered}
\mathrm{ER}_{\mathrm{MT}}=\mathrm{BMR} \times 1.4 \times 0.25 \\
\mathrm{ER}_{\mathrm{WL}}=\mathrm{BMR} \times 1 \times 0.25
\end{gathered}
$$

Table 1. Composition of the high-protein and normal-protein test drinks per $100 \mathrm{~mL}$ compared to whole milk.

\begin{tabular}{ccccc}
\hline \multicolumn{2}{c}{ Product (per $\mathbf{1 0 0} \mathbf{~ m L )}$} & HP Drink & NP Drink & Whole Milk \\
\hline Total Energy & $(\mathrm{kcal})$ & 130 & 130 & 63 \\
\hline \multirow{4}{*}{ Protein } & $(\mathrm{g})$ & 10.0 & 5.0 & 3.4 \\
& Energy (\%) & 30.7 & 15.3 & 21.9 \\
\multirow{4}{*}{ Fat } & Casein (g) & 8.0 & 4.0 & 2.7 \\
& Whey (g) & 2.0 & 1.0 & 0.7 \\
Carbohydrate & $(\mathrm{g})$ & 3.5 & 3.5 & 3.6 \\
& Energy (\%) & 24.2 & 24.2 & 50.6 \\
& Energy (\%) & 14.7 & 19.7 & 4.6 \\
& Lactose (g) & 45.1 & 60.5 & 27.9 \\
\hline
\end{tabular}

HP: high-protein test drink, NP: normal-protein test drink.

The quantity of each drink to be served was calculated considering the energy density of the drinks. The energy density of the drinks in $\mathrm{kcal} / 100 \mathrm{~mL}\left(\mathrm{ED}_{\mathrm{kcal} / 100 \mathrm{~mL}}\right)$ was:

$$
\mathrm{ED}_{\mathrm{kcal} / 100 \mathrm{~mL}}=130 \mathrm{kcal} / 100 \mathrm{~mL}
$$

The physical density (d) of the drinks was $1.088 \mathrm{~kg} / \mathrm{L}$. Therefore, the energy density of the drinks in $\mathrm{kcal} / 100 \mathrm{~g}\left(\mathrm{ED}_{\mathrm{kcal} / 100 \mathrm{~g}}\right)$ was:

$$
\begin{gathered}
\mathrm{ED}_{\mathrm{kcal} / 100 \mathrm{~g}}=\left(\mathrm{ED}_{\mathrm{kcal} / 100 \mathrm{~mL}} \div \mathrm{d} \times 100\right) \times 100 \\
\mathrm{ED}_{\mathrm{kcal} / 100 \mathrm{~g}}=130 \div 1.09=119 \mathrm{kcal} / 100 \mathrm{~g}
\end{gathered}
$$

Finally, the quantity of drink to be served was as follows:

$$
\text { Quantity }(\mathrm{g})=\left(\mathrm{ER} \div \mathrm{ED}_{\mathrm{kcal} / 100 \mathrm{~g}}\right) \times 100=(\mathrm{ER} \div 119) \times 100
$$

Each participant consumed the four different types of test drink: normal-protein weight loss (NPWL), normal-protein weight maintenance (NPMT), high-protein weight loss (HPWL) and high-protein weight maintenance (HPMT) in a randomized order and at a standardized time. The composition of the test drinks was double-blinded and the drinks were labeled A, B, C and D. Nutricia labeled the drinks and generated the random allocation sequence. The drinks were weighed to the nearest gram and placed into neutral sealed cups with a straw. Participants were required to consume at least $80 \%$ of each drink and failure to do so would result in their withdrawal from the study. The composition of the drinks was unblinded to the researchers after the final participant completed the study.

\subsection{Ad Libitum EI}

The ad libitum buffet-style test meal consisted of a counter-balanced selection of 25 sweet and savory, high- and low-calorie food and drink items, all of which were provided in excess (Table S2). All food and drink items were chosen to be commercially available in the UK and Greece. Buffet items were offered either in transparent plastic containers or in their original packaging. The buffet was 
provided $120 \mathrm{~min}$ after test drink consumption, participants were given access to the buffet for $30 \mathrm{~min}$ and instructed to consume as much or as little of each buffet item as they wanted until they were satisfied. All foods and drinks were presented identically on each occasion and covertly weighed before and after the buffet. Ad libitum energy and macronutrient intakes were calculated using nutritional values provided by the manufacturer, or by using an electronic version of McCance and Widdowson's The Composition of Foods [32]; NETWISP ${ }^{\text {тм }}$ software (version 3.0 for Windows, Tinuviel Software, Anglesey, UK).

\subsection{Subjective Appetite Assessment}

Appetite perceptions (hunger, fullness and prospective food consumption (PFC)) were measured in adult and elderly participants using previously validated $100 \mathrm{~mm}$ visual analog scales (VASs, [33]). Participants indicated their subjective feelings of appetite by marking a vertical line on the VAS. A composite appetite score was calculated at each time of measurement using the following formula:

$$
[\text { Hunger }+(100-\text { fullness })+\text { prospective consumption }] / 3
$$

Higher composite appetite scores relate to elevated feelings of appetite. The composite appetite score is increasingly used in the literature for ease of data analysis and presentation [25,34].

Children and adolescents used a 9-point Likert scale to rate fullness (How full do you feel?) and PFC (How much do you think you could eat now?), with 1 representing "Not at all full"/"Nothing at all" and 9 representing "As full as I've ever felt"/"A large amount" for fullness and PFC, respectively. Participants were not permitted to view their previous ratings when completing the scales.

\subsection{Test Drink Pleasantness and Satisfaction}

After consuming the test drinks, participants rated the drinks for pleasantness and satisfaction. The adult and elderly participants used a $100 \mathrm{~mm}$ VAS to rate the drinks, with "Not at all pleasant" /"Not at all satisfying" on the left side and "Extremely pleasant" /"Very satisfying" on the right side of the pleasantness and satisfaction scales. Children and adolescents used a 9-point Likert scale adapted from Jansen et al. [35] to rate the drinks for pleasantness and satisfaction. The scale consisted of 5 cartoon faces (smileys). The first cartoon face on the left (unhappy) reflecting low perceived pleasantness/satisfaction was scored 1 and the last face (very happy) on the right of the scale, reflecting high perceived pleasantness/satisfaction, was scored 9. Participants could rate in between two faces, creating a 9-point scale.

\subsection{Food Reward: Leeds Food Preference Questionnaire (LFPQ)}

The Leeds Food Preference Questionnaire [36] provided a baseline measure of liking and wanting along dimensions of fat and taste. Participants were presented with an array of pictures of individual food items common in the diet. Foods were chosen by the local research team from a validated database to be either predominantly high ( $>50 \%$ energy) or low ( $<20 \%$ energy) in fat, sweet or savory in taste, but similar in familiarity, protein content and cultural suitability for the study population [37]. The LFPQ has been validated in previous studies investigating dietary protein [38-40]. Explicit liking was measured by participants rating the extent to which they liked each food using a $100 \mathrm{~mm}$ VAS ("How pleasant would it be to taste this food now?"). Implicit wanting was assessed using a forced choice methodology so that every image from each of the four food types was compared to every other type over 96 trials (food pairs). Reaction times for all responses were covertly recorded for each food type after adjusting for frequency of selection [37]. Fat bias scores for liking and wanting were calculated as the difference between the high-fat scores and the low-fat scores. Sweet bias scores were calculated as the difference between the sweet and savory scores. Positive values indicated greater liking/wanting for high fat $>$ low fat or sweet $>$ savory and negative values indicated the reverse. 


\subsection{Blood Sampling and Processing}

At all test visits, glucose, insulin, total ghrelin, PYY and GLP-1 were measured fasted and postprandially (at 30,60 and 120 min after eating), while leptin was measured at the first test visit only. An intravenous cannula (BD Venflon, BD, UK) was inserted into an antecubital vein for the collection of venous blood samples. During the trials, the cannula was kept patent with $2 \mathrm{~mL}$ flushes of $0.9 \% \mathrm{NaCl}(\mathrm{aq})$ isotonic saline solution (Baxter Healthcare, UK) after each bloodletting. At each time point, a venous blood sample was collected into a $4.9 \mathrm{~mL}$ EDTA-coated monovette (S-Monovette, Sarstedt, Nümbrecht, Germany) for the measurement of plasma total ghrelin, PYY and GLP-1 concentrations. A second venous blood sample was collected into a $2.7 \mathrm{~mL}$ lithium heparin-coated monovette (S-Monovette, Sarstedt, Nümbrecht, Germany) for the measurement in plasma of leptin, glucose and insulin. Immediately after blood collection, collection tubes were placed in ice and $160 \mu \mathrm{L}$ of a preservative containing 4-(2-aminoethyl) benzenesulfonylfluoride hydrochloride (Roche, Basel, Switzerland), dipeptidyl peptidase-4 inhibitor (Merck Millipore, Darmstadt, Germany) and protease inhibitor cocktail (Sigma-Aldrich, St. Louis, MO, USA) were added to EDTA-coated monovettes. After gentle inversion, both monovettes were spun at $1000 \mathrm{~g}$ for $15 \mathrm{~min}$ in a centrifuge at $4{ }^{\circ} \mathrm{C}$ and plasma was stored at $-80^{\circ} \mathrm{C}$ for batch analysis at the conclusion of the study. Identical blood sampling and processing procedures were followed at ABDN and HUA. Blood samples were collected from adult and elderly participants only. No samples were collected from children, as gaining ethical approval for blood samples in this vulnerable group was challenging.

\subsection{Biomarker Analysis}

\subsubsection{Appetite Hormones}

Total ghrelin concentrations were measured using a human-specific radioimmunoassay kit (Linco Research, St. Charles, MO, USA) at the laboratory of JJ Holst. The lowest concentration of ghrelin detectable using this assay was $93 \mathrm{pg} / \mathrm{mL}$. The limit of linearity for this assay was $6000 \mathrm{pg} / \mathrm{mL}$. All samples were read using a gamma counter. The between- and within-volunteer CVs were 39\% and $14 \%$, respectively. Total PYY and GLP-1 were measured in duplicate using an electrochemical luminescence immunoassay kit (Meso Scale Discovery, Rockville, Maryland, USA) on the Meso Scale Discovery ${ }^{\circledR}$ multiarray assay platform (Meso Scale Discovery, Rockville, Maryland, USA) at the Core Biomedical Assay Laboratory (CBAL), Cambridge. The PYY immunoassay measured both PYY 1-36 and $\mathrm{PYY}_{3-36}$ with a range of 30-3000 pg/mL. Inter-assay CVs of 7.8-16.4\% were obtained. The GLP-1 immunoassay measures all endogenous forms of GLP-1 (including GLP-11-36, GLP-11-37, GLP-17-36, GLP-17-37, GLP-19-36 and GLP-19-37) and has a range of 1.4-1000 pg/mL and CVs of 5.2-8.2\% for most of the analytical range. Leptin analysis was performed at CBAL using an in-house two-site DELFIA ${ }^{\circledR}$ assay, which used a monoclonal capture antibody and a polyclonal detection antibody with fluorescent detection using europium-labeled streptavidin [41,42]. The antibodies and standards were sourced from R\&D Systems (R\&D Systems Europe, Abingdon, UK). This assay had a lower limit of detection of $0.1 \mathrm{ng} / \mathrm{mL}$ and intra-assay CVs of 3.9-7.1\%.

\subsubsection{Glucose Homeostasis}

Glucose and insulin plasma analysis was conducted at the University of Aberdeen, Rowett Institute, Technical Services department. Glucose concentrations were measured using a hexokinase method on a Dimension ${ }^{\circledR}$ clinical chemistry analyzer (Siemens Healthcare GmbH, Erlangen, Germany) with CVs of $<2 \%$ within the reference range. Insulin was detected using a Liaison ${ }^{\circledR}$ XL automated immunoassay analyzer (DiaSorin, Italy) with a chemiluminescence immunoassay, which had a range of 20-3470 pmol/L and intra-assay CVs of 5.0-6.0\% across the analytical range. The homeostatic model assessment [43] was used to estimate hepatic insulin resistance (HOMA-IR):

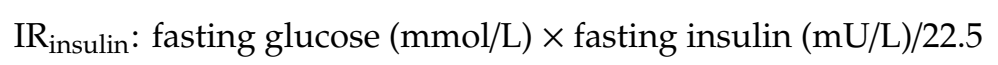


$\beta$-cell function was measured using an early insulin secretion function index (insulinogenic index (IGI)):

$$
\text { IGI: (Insulin_0 min - Insulin_30 min)/(Glucose_0 min - Glucose_30 min) }
$$

Insulin to glucose ratio (IGR) was also calculated.

\subsection{Statistical Analysis}

It was calculated that 16 participants in each group (defined by age, BMI, gender and study site) would give approximately $80 \%$ power to detect group and treatment differences in any variable comparable to the unpredictable variation between groups or within individuals, i.e., to detect a standard effect size of approximately 1.0. Main factor effect comparisons are based on combinations of groups and so larger volunteer numbers had the power to detect smaller effect sizes.

Variables were analyzed by linear mixed models using residual maximum likelihood with random effect terms for volunteer and fixed effect terms for test drink type, age group, BMI group, gender, site and all two-way and three-way interactions. An additional analysis was carried out in each case in which the drink effect was decomposed into its factorial components of composition (HP vs. NP) and quantity (WL vs. MT). Where data were collected at several timepoints in a day (appetite scores and gut hormones), an additional random effect term for day, and fixed effect term for time, were included in the models. Significance of fixed effect terms was assessed by F statistics calculated from Wald statistics, with estimated denominator degrees of freedom. Drinks were compared with post hoc tests based on least significant differences. A $p$ value $<0.05$ was considered to indicate statistical significance. Analyses were carried out using Genstat v17 (VSN International, Hemel Hempstead, UK). Data are presented as mean \pm the standard error of the differences of the mean (SED) unless stated otherwise.

\section{Results}

\subsection{Participants}

In total, 424 members of the public were enrolled in the study (See the Consolidated Standards of Reporting Trials (CONSORT) flow diagram (Figure S1) summarizing the participant flow). Thirty-three participants discontinued the study after randomization, of which five were excluded as they consumed $<80 \%$ of at least one of the test drinks. Therefore, 391 participants across ABDN and HUA completed the study, as 103 children, 109 adolescents, 97 adults and 82 elderly. The characteristics of the participants from ABDN and HUA who completed the study are presented in Table 2. In addition, Supplementary Materials Table S3 presents the number of participants allocated to each group at both sites. 
Table 2. Participant characteristics by age group and site ${ }^{1}$.

\begin{tabular}{|c|c|c|c|c|c|c|c|c|c|c|c|c|}
\hline & \multicolumn{3}{|c|}{ Children } & \multicolumn{3}{|c|}{ Adolescents } & \multicolumn{3}{|c|}{ Adults } & \multicolumn{3}{|c|}{ Elderly } \\
\hline & $\begin{array}{l}\text { ABDN } \\
(n=39)\end{array}$ & $\begin{array}{c}\text { HUA } \\
(n=64)\end{array}$ & $\begin{array}{c}\text { All } \\
(n=103)\end{array}$ & $\begin{array}{l}\text { ABDN } \\
(n=45)\end{array}$ & $\begin{array}{c}\text { HUA } \\
(n=64)\end{array}$ & $\begin{array}{c}\text { All } \\
(n=109)\end{array}$ & $\begin{array}{l}\text { ABDN } \\
(n=46)\end{array}$ & $\begin{array}{c}\text { HUA } \\
(n=51)\end{array}$ & $\begin{array}{c}\text { All } \\
(n=97)\end{array}$ & $\begin{array}{l}\text { ABDN } \\
(n=36)\end{array}$ & $\begin{array}{c}\text { HUA } \\
(n=46)\end{array}$ & $\begin{array}{c}\text { All } \\
(n=82)\end{array}$ \\
\hline Age (years) & $8.72 \pm 0.69$ & $9.20 \pm 0.65$ & $9.02 \pm 0.70$ & $15.4 \pm 1.28$ & $14.5 \pm 1.33$ & $14.9 \pm 1.37$ & $29.9 \pm 7.34$ & $32.8 \pm 6.71$ & $31.4 \pm 7.13$ & $68.0 \pm 3.82$ & $68.5 \pm 3.88$ & $68.3 \pm 3.84$ \\
\hline Height (m) & $1.37 \pm 0.08$ & $1.40 \pm 0.06$ & $1.39 \pm 0.07$ & $1.65 \pm 0.09$ & $1.67 \pm 0.09$ & $1.66 \pm 0.09$ & $1.71 \pm 0.09$ & $1.71 \pm 0.10$ & $1.71 \pm 0.09$ & $1.64 \pm 0.08$ & $1.62 \pm 0.08$ & $1.62 \pm 0.08$ \\
\hline Weight (kg) & $31.6 \pm 6.94$ & $38.3 \pm 8.39$ & $35.7 \pm 8.49$ & $60.3 \pm 12.3$ & $65.7 \pm 10.4$ & $63.4 \pm 11.5$ & $71.0 \pm 14.2$ & $76.1 \pm 14.4$ & $73.7 \pm 14.5$ & $68.6 \pm 11.9$ & $75.7 \pm 14.4$ & $72.6 \pm 13.7$ \\
\hline BMI $\left(\mathrm{kg} / \mathrm{m}^{2}\right)$ & $16.7 \pm 2.41$ & $19.5 \pm 3.33$ & $18.4 \pm 3.31$ & $22.2 \pm 4.23$ & $23.5 \pm 2.82$ & $23.0 \pm 3.51$ & $24.3 \pm 4.07$ & $25.9 \pm 4.33$ & $25.1 \pm 4.27$ & $25.5 \pm 3.58$ & $28.9 \pm 4.71$ & $27.4 \pm 4.55$ \\
\hline $\mathrm{BMR}^{2}(\mathrm{MJ})$ & $4.93 \pm 0.62$ & $5.52 \pm 0.82$ & $5.30 \pm 0.8$ & $6.70 \pm 1.02$ & $7.14 \pm 1.04$ & $6.96 \pm 1.05$ & $6.67 \pm 1.04$ & $7.18 \pm 1.17$ & $6.94 \pm 1.13$ & $5.73 \pm 0.74$ & $6.54 \pm 1.00$ & $6.19 \pm 0.98$ \\
\hline Body fat ${ }^{3}(\%)$ & $22.8 \pm 5.70$ & $25.4 \pm 5.93$ & $24.4 \pm 5.95$ & $24.8 \pm 8.47$ & $24.8 \pm 7.54$ & $24.8 \pm 7.90$ & $23.6 \pm 10.1$ & $26.2 \pm 9.95$ & $24.9 \pm 10.1$ & $32.4 \pm 6.78$ & $35.4 \pm 6.78$ & $34.2 \pm 6.90$ \\
\hline Waist Circumference ${ }^{4}(\mathrm{~cm})$ & $64.5 \pm 8.56$ & $71.8 \pm 12.4$ & $69.6 \pm 11.8$ & $81.6 \pm 10.9$ & $84.9 \pm 9.75$ & $83.7 \pm 10.3$ & $91.4 \pm 13.0$ & $96.9 \pm 12.4$ & $94.4 \pm 12.9$ & $100 \pm 14.0$ & $109 \pm 12.8$ & $106 \pm 14.0$ \\
\hline Visceral Fat ${ }^{4}(\%)$ & $3.67 \pm 2.23$ & $4.95 \pm 3.25$ & $4.58 \pm 3.03$ & $5.43 \pm 3.67$ & $6.05 \pm 3.28$ & $5.81 \pm 3.43$ & $7.72 \pm 4.92$ & $9.64 \pm 4.64$ & $8.76 \pm 4.84$ & $10.4 \pm 4.25$ & $15.1 \pm 6.76$ & $13.2 \pm 6.29$ \\
\hline
\end{tabular}

${ }^{1}$ Values are means \pm SD; ${ }^{2}$ calculated by Schofield equation; ${ }^{3}$ measured by whole body BIA ${ }^{4}$ measured by abdominal VISCAN bio-impedance; ABDN: Scotland, BIA: bioelectrical impedance analysis, BMR: basal metabolic rate, HUA: Greece. 


\subsection{Test Drinks}

The average test drink energy ( $\mathrm{kcal})$ and protein $(\mathrm{g})$ consumption, corrected for mass consumed, varied significantly between age groups $(p<0.001$; Table 3$)$.

Table 3. Test drink energy and protein consumption ${ }^{1}$.

\begin{tabular}{|c|c|c|c|c|c|c|c|}
\hline & & NPWL & NPMT & HPWL & HPMT & SED $_{\text {type }}$ & $p_{\text {type }}{ }^{2}$ \\
\hline \multirow{4}{*}{ 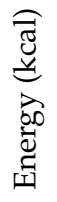 } & Children $(n=102)$ & 306 & 425 & 304 & 425 & \multirow{4}{*}{10} & \multirow{4}{*}{$<0.001$} \\
\hline & $\begin{array}{l}\text { Adolescents } \\
\quad(n=108)\end{array}$ & 406 & 565 & 402 & 557 & & \\
\hline & Adults $(n=97)$ & 399 & 553 & 395 & 548 & & \\
\hline & Elderly $(n=82)$ & 348 & 483 & 343 & 478 & & \\
\hline \multirow{4}{*}{ 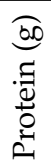 } & Children $(n=102)$ & 11.4 & 15.9 & 23.3 & 32.6 & \multirow{4}{*}{0.6} & \multirow{4}{*}{$<0.001$} \\
\hline & Adolescents $(n=108)$ & 15.2 & 21.1 & 30.8 & 42.7 & & \\
\hline & Adults $(n=97)$ & 14.9 & 20.6 & 30.3 & 42.0 & & \\
\hline & Elderly $(n=82)$ & 13.0 & 18.0 & 26.3 & 36.6 & & \\
\hline
\end{tabular}

1 Corrected for mass consumed. Mean data are presented for drink type (NPWL, NPMT, HPWL, HPMT);

2 determined by ANOVA, differences are statistically significant when $p<0.05$; HPMT: high-protein maintenance, HPWL: high-protein weight loss, NPMT: normal-protein maintenance, NPWL: normal-protein weight loss.

\subsection{Ad Libitum EI}

Differences in mean ad libitum EI after the test drinks between age groups, weight categories, genders and sites are presented in Table 4 . Differences between age groups in response to the quantity of drink provided (WL vs. MT) approached significance $(p=0.074)$.

Table 4. Ad libitum EI (kcal) ${ }^{1}$.

\begin{tabular}{|c|c|c|c|c|c|c|c|c|c|c|c|c|c|c|c|}
\hline & Group & NPWL & NPMT & HPWL & HPMT & SED type & $p_{\text {type }}{ }^{2}$ & NP & HP & SED $_{\text {composition }}$ & $p_{\text {composition }}{ }^{2}$ & WL & MT & SED quantity & $p_{\text {quantity }}{ }^{2}$ \\
\hline \multirow{4}{*}{$\stackrel{8}{<}$} & $\begin{array}{l}\text { Children } \\
(n=103)\end{array}$ & 718 & 641 & 679 & 646 & \multirow{4}{*}{29} & \multirow{4}{*}{0.483} & 679 & 663 & \multirow{4}{*}{21} & \multirow{4}{*}{0.941} & 699 & 644 & \multirow{4}{*}{21} & \multirow{4}{*}{0.074} \\
\hline & $\begin{array}{l}\text { Adolescents } \\
(n=109)\end{array}$ & 950 & 876 & 940 & 852 & & & 914 & 895 & & & 945 & 864 & & \\
\hline & $\begin{array}{l}\text { Adults } \\
(n=97)\end{array}$ & 672 & 603 & 641 & 627 & & & 639 & 634 & & & 658 & 615 & & \\
\hline & $\begin{array}{l}\text { Elderly } \\
(n=82)\end{array}$ & 526 & 522 & 478 & 500 & & & 524 & 490 & & & 502 & 510 & & \\
\hline \multirow[t]{2}{*}{$\sum_{m}^{E}$} & $\begin{array}{c}\text { Normal } \\
\text { Weight } \\
(n=221)\end{array}$ & 701 & 644 & 656 & 636 & \multirow[t]{2}{*}{21} & \multirow[t]{2}{*}{0.928} & 672 & 646 & \multirow[t]{2}{*}{15} & \multirow[t]{2}{*}{0.700} & 677 & 639 & \multirow[t]{2}{*}{15} & \multirow[t]{2}{*}{0.858} \\
\hline & $\begin{array}{c}\text { Overweight } \\
(n=170)\end{array}$ & 732 & 677 & 715 & 675 & & & 706 & 696 & & & 725 & 677 & & \\
\hline \multirow{2}{*}{$\frac{\tilde{\Xi}}{\tilde{E}}$} & $\begin{array}{c}\text { Males } \\
(n=171)\end{array}$ & 825 & 751 & 773 & 744 & \multirow[t]{2}{*}{21} & \multirow[t]{2}{*}{0.166} & 789 & 758 & \multirow[t]{2}{*}{15} & \multirow[t]{2}{*}{0.220} & 799 & 749 & \multirow[t]{2}{*}{15} & \multirow[t]{2}{*}{0.123} \\
\hline & $\begin{array}{l}\text { Females } \\
(n=220)\end{array}$ & 608 & 569 & 598 & 567 & & & 589 & 581 & & & 603 & 567 & & \\
\hline \multirow[t]{2}{*}{ 苛 } & $\begin{array}{c}\text { HUA } \\
(n=225)\end{array}$ & 806 & 766 & 797 & 756 & \multirow[t]{2}{*}{21} & \multirow[t]{2}{*}{0.245} & 787 & 778 & \multirow[t]{2}{*}{15} & \multirow[t]{2}{*}{0.272} & 801 & 761 & \multirow[t]{2}{*}{15} & \multirow[t]{2}{*}{0.897} \\
\hline & $\begin{array}{c}\text { ABDN } \\
(n=166)\end{array}$ & 627 & 555 & 572 & 555 & & & 591 & 565 & & & 600 & 555 & & \\
\hline
\end{tabular}

${ }^{1} n=391$. Mean data are presented for drink type (NPWL, NPMT, HPWL, HPMT), drink composition (NP, HP) and drink quantity (WL, MT); ${ }^{2}$ determined by ANOVA (with age, BMI, gender and site as fixed factors), differences are statistically significant when $p<0.05$; ABDN: Scotland, EI: energy intake, HP: high protein, HPMT: high-protein maintenance, HPWL: high-protein weight loss, HUA: Greece, MT: weight maintenance, NP: normal protein, NPMT: normal-protein maintenance, NPWL: normal-protein weight loss, WL: weight loss.

There were no significant differences between weight categories, genders or sites. Furthermore, there were no significant differences in total caloric intake (test drink EI + ad libitum EI) between age groups, weight categories, genders or sites in response to drink type, composition or quantity (Table 5). 
Table 5. Total caloric intake (kcal) ${ }^{1}$.

\begin{tabular}{|c|c|c|c|c|c|c|c|c|c|c|c|c|c|c|c|}
\hline & Group & NPWL & NPMT & HPWL & HPMT & SED $_{\text {type }}$ & $p_{\text {type }}{ }^{2}$ & $\mathrm{NP}$ & $\mathrm{HP}$ & $\mathrm{SED}_{\text {composition }}$ & $p_{\text {composition }}{ }^{2}$ & WL & MT & SED $_{\text {quantity }}$ & $p_{\text {quantity }}{ }^{2}$ \\
\hline \multirow{4}{*}{$\stackrel{0}{\circ}$} & $\begin{array}{l}\text { Children } \\
(n=103)\end{array}$ & 1022 & 1075 & 991 & 1077 & \multirow{4}{*}{50} & \multirow{4}{*}{0.562} & 1049 & 1034 & \multirow{4}{*}{46} & \multirow{4}{*}{0.793} & 1007 & 1076 & \multirow{4}{*}{46} & \multirow{4}{*}{0.143} \\
\hline & $\begin{array}{l}\text { Adolescents } \\
\quad(n=109)\end{array}$ & 1372 & 1461 & 1352 & 1416 & & & 1416 & 1384 & & & 1362 & 1438 & & \\
\hline & $\begin{array}{l}\text { Adults } \\
(n=97)\end{array}$ & 1078 & 1168 & 1048 & 1186 & & & 1123 & 1117 & & & 1063 & 1177 & & \\
\hline & $\begin{array}{l}\text { Elderly } \\
(n=82)\end{array}$ & 885 & 1022 & 835 & 993 & & & 953 & 914 & & & 860 & 1008 & & \\
\hline \multirow[t]{2}{*}{$\sum_{\infty}$} & $\begin{array}{c}\text { Normal } \\
\text { Weight } \\
(n=221)\end{array}$ & 1235 & 1325 & 1,85 & 1314 & \multirow[t]{2}{*}{31} & \multirow[t]{2}{*}{0.625} & 1280 & 1249 & \multirow[t]{2}{*}{29} & \multirow[t]{2}{*}{0.499} & 1210 & 1319 & \multirow[t]{2}{*}{29} & \multirow[t]{2}{*}{0.940} \\
\hline & $\begin{array}{c}\text { Overweight } \\
(n=170)\end{array}$ & 943 & 1038 & 928 & 1023 & & & 991 & 975 & & & 936 & 1030 & & \\
\hline \multirow{2}{*}{ 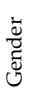 } & $\begin{array}{c}\text { Males } \\
(n=171)\end{array}$ & 1044 & 1126 & 996 & 1112 & \multirow[t]{2}{*}{31} & \multirow[t]{2}{*}{0.775} & 1085 & 1054 & \multirow[t]{2}{*}{29} & \multirow[t]{2}{*}{0.751} & 1020 & 1119 & \multirow[t]{2}{*}{29} & \multirow[t]{2}{*}{0.455} \\
\hline & $\begin{array}{l}\text { Females } \\
(n=220)\end{array}$ & 1134 & 1237 & 1117 & 1225 & & & 1185 & 1171 & & & 1125 & 1231 & & \\
\hline \multirow[t]{2}{*}{ 苟 } & $\begin{array}{c}\text { HUA } \\
(n=225)\end{array}$ & 995 & 1075 & 942 & 1063 & \multirow[t]{2}{*}{30} & \multirow[t]{2}{*}{0.287} & 1035 & 1002 & 28 & 0.239 & 968 & 1069 & \multirow[t]{2}{*}{28} & \multirow[t]{2}{*}{0.862} \\
\hline & $\begin{array}{c}\text { ABDN } \\
(n=166)\end{array}$ & 1183 & 1288 & 1171 & 1274 & & & 1236 & 1222 & & & 1177 & 1281 & & \\
\hline
\end{tabular}

The data for mean ad libitum energy and macronutrient intake with all participants combined are reported in Table S4, to explore drink effects. Ad libitum EI was significantly greater after consuming the NPWL drink, in comparison to the other drink types $(p<0.001)$. There were small but statistically significant differences in energy and macronutrient intakes between drinks fed at WL or MT quantities, reflected by higher intakes after WL (all $p<0.001$ ). There were no differences in ad libitum energy or nutrient intakes between the NP and HP drinks.

Visit number had a significant effect on ad libitum EI, with EI significantly greater for visits 2, 3 and 4 compared to visit 1 for all participants combined (Supplementary Materials Table S5; $p=0.001$ ). In addition, the effect of visit number on ad libitum EI differed significantly between age groups $(p<0.001)$.

\subsection{Subjective Appetite Assessment}

Table 6 presents the fullness and PFC ratings for drink type, composition and quantity $\mathrm{x}$ time interactions for children and adolescents. Fullness did not differ between children and adolescents in response to drink type $(p=0.252)$, composition $(p=0.220)$ or quantity $(p=0.554)$. There were no significant differences in ratings of PFC in response to drink type $(p=0.332)$, composition $(p=0.209)$ or quantity $(p=0.653)$ when comparing children and adolescents. There were no significant differences in fullness or PFC ratings between weight categories, genders or sites (data not shown).

Table 7 presents the composite appetite score for drink type, composition and quantity $\mathrm{x}$ time interactions for adults and elderly. The adults' appetite score was significantly greater than that of the elderly across all drink types $(p<0.004)$ and in response to both drink quantities $(p<0.001)$. There were no significant differences between adult and elderly appetite scores in response to drink composition $(p=0.624)$. There were no significant differences in composite appetite scores between weight categories, genders or sites (data not shown). 
Table 6. Children ${ }^{1}$ and adolescent ${ }^{2}$ motivation to eat at baseline and in response to test drink type, composition and quantity.

\begin{tabular}{|c|c|c|c|c|c|c|c|c|c|c|c|c|c|c|c|c|}
\hline & & \multirow[t]{2}{*}{ Time (mins) } & \multirow[t]{2}{*}{ NPWL } & \multirow[t]{2}{*}{ NPMT } & \multirow[t]{2}{*}{ HPWL } & \multirow[t]{2}{*}{ HPMT } & \multirow[t]{2}{*}{$\mathrm{SED}_{\text {type }}$} & \multirow{2}{*}{$\begin{array}{c}\begin{array}{r}\text { Type.Time } \\
\text { Interaction }\end{array} \\
p^{3}\end{array}$} & \multirow[t]{2}{*}{ NP } & \multirow[t]{2}{*}{ HP } & \multirow[t]{2}{*}{$\mathrm{SED}_{\text {composition }}$} & \multirow{2}{*}{$\begin{array}{c}\begin{array}{c}\text { Composition.Time } \\
\text { Interaction }\end{array} \\
p^{3} \\
\end{array}$} & \multirow[t]{2}{*}{ WL } & \multirow[t]{2}{*}{ MT } & \multirow[t]{2}{*}{ SED $_{\text {quantity }}$} & \multirow{2}{*}{$\begin{array}{c}\text { Quantity.Time } \\
\text { Interaction }\end{array}$} \\
\hline & & & & & & & & & & & & & & & & \\
\hline \multirow{10}{*}{ 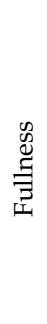 } & & 0 & 2.25 & 2.02 & 1.77 & 2.19 & \multirow{5}{*}{0.21} & \multirow{5}{*}{0.252} & 2.13 & 1.98 & \multirow{5}{*}{0.16} & \multirow{5}{*}{0.220} & 2.01 & 2.10 & \multirow{5}{*}{0.16} & \multirow{5}{*}{0.554} \\
\hline & ฮี & 30 & 3.73 & 3.86 & 3.37 & 3.71 & & & 3.79 & 3.54 & & & 3.55 & 3.78 & & \\
\hline & $\frac{7}{7}$ & 60 & 3.06 & 3.14 & 3.02 & 2.83 & & & 3.10 & 2.92 & & & 3.04 & 2.98 & & \\
\hline & تี & 90 & 2.60 & 2.75 & 2.52 & 2.51 & & & 2.68 & 2.51 & & & 2.56 & 2.63 & & \\
\hline & 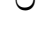 & 120 & 2.13 & 2.28 & 2.33 & 2.19 & & & 2.21 & 2.26 & & & 2.23 & 2.23 & & \\
\hline & \pm & 0 & 2.69 & 2.90 & 2.95 & 2.95 & \multirow{5}{*}{0.18} & \multirow{15}{*}{0.332} & 2.80 & 2.95 & \multirow{5}{*}{0.14} & & 2.82 & 2.92 & \multirow{5}{*}{0.14} & \multirow{15}{*}{0.653} \\
\hline & $\overrightarrow{\tilde{J}}$ & 30 & 4.49 & 4.85 & 5.06 & 5.13 & & & 4.67 & 5.10 & & & 4.78 & 4.99 & & \\
\hline & 过 & 60 & 4.15 & 4.44 & 4.52 & 4.59 & & & 4.29 & 4.56 & & & 4.33 & 4.52 & & \\
\hline & $\frac{0}{0}$ & 90 & 3.65 & 3.94 & 3.91 & 4.06 & & & 3.80 & 3.98 & & & 3.78 & 4.00 & & \\
\hline & $\frac{8}{4}$ & 120 & 3.28 & 3.53 & 3.59 & 3.59 & & & 3.40 & 3.59 & & & 3.43 & 3.56 & & \\
\hline \multirow{10}{*}{ 壵 } & & 0 & 6.61 & 6.89 & 7.05 & 7.16 & \multirow{5}{*}{0.19} & & 6.75 & 7.10 & & & 6.83 & 7.02 & & \\
\hline & $\widetilde{్}$ & 30 & 6.00 & 6.00 & 6.22 & 5.90 & & & 6.00 & 6.06 & & & 6.11 & 5.95 & & \\
\hline & $\bar{t}$ & 60 & 6.70 & 6.55 & 6.54 & 6.96 & & & 6.62 & 6.75 & 0.16 & 0.209 & 6.62 & 6.76 & 0.16 & \\
\hline & בี & 90 & 7.14 & 7.14 & 7.05 & 7.26 & & & 7.14 & 7.16 & & & 7.10 & 7.20 & & \\
\hline & & 120 & 7.62 & 7.65 & 7.55 & 7.60 & & & 7.64 & 7.57 & & & 7.58 & 7.63 & & \\
\hline & s & 0 & 6.19 & 6.06 & 6.05 & 5.90 & \multirow{5}{*}{0.21} & & 6.13 & 5.98 & & & 6.12 & 5.98 & & \\
\hline & 䒕 & 30 & 5.08 & 4.63 & 4.58 & 4.31 & & & 4.85 & 4.44 & & & 4.83 & 4.47 & & \\
\hline & 过 & 60 & 5.47 & 5.05 & 5.08 & 4.96 & & & 5.26 & 5.02 & 0.14 & & 5.27 & 5.01 & 0.14 & \\
\hline & 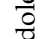 & 90 & 5.85 & 5.66 & 5.61 & 5.61 & & & 5.76 & 5.61 & & & 5.73 & 5.64 & & \\
\hline & 8 & 120 & 6.42 & 6.08 & 6.07 & 6.10 & & & 6.25 & 6.09 & & & 6.25 & 6.09 & & \\
\hline
\end{tabular}

${ }^{1} n=103$ for children ${ }^{2} n=109$ for adolescents; ${ }^{3}$ determined by ANOVA between age groups, differences are statistically significant when $p<0.05$. Mean data are presented for drink type (NPWL, NPMT, HPWL, HPMT), drink composition (NP, HP) and drink quantity (WL, MT); HP: high protein, HPMT: high-protein maintenance, HPWL: high-protein weight loss, MT: weight maintenance, NP: normal protein, NPMT: normal-protein maintenance, NPWL: normal-protein weight loss, PFC: prospective food consumption, WL: weight loss. 
Table 7. Adult ${ }^{1}$ and elderly ${ }^{2}$ composite appetite scores at baseline and in response to test drink type, composition and quantity.

\begin{tabular}{|c|c|c|c|c|c|c|c|c|c|c|c|c|c|c|c|}
\hline & Time (mins) & NPWL & NPMT & HPWL & HPMT & $\mathrm{SED}_{\text {type }}$ & $\begin{array}{l}\text { Type.Time } \\
\text { Interaction }\end{array}$ & NP & HP & SED composition & $\begin{array}{l}\text { Composition.Time } \\
\text { Interaction }\end{array}$ & WL & MT & SED $_{\text {quantity }}$ & $\begin{array}{l}\text { Quantity.Time } \\
\text { Interaction }\end{array}$ \\
\hline & & & & & & & $p^{3}$ & & & & $p^{3}$ & & & & $p^{3}$ \\
\hline \multirow{10}{*}{ 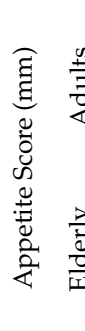 } & 0 & 63.2 & 65.6 & 61.5 & 62.8 & \multirow{5}{*}{2.1} & \multirow{10}{*}{0.004} & 64.4 & 62.1 & & \multirow{10}{*}{0.624} & 62.3 & 64.2 & \multirow{5}{*}{1.6} & \multirow{9}{*}{$<0.001$} \\
\hline & 30 & 42.6 & 35.5 & 41.2 & 34.8 & & & 39.0 & 38.0 & & & 41.9 & 35.1 & & \\
\hline & 60 & 46.7 & 40.3 & 44.6 & 37.9 & & & 43.5 & 41.3 & 1.6 & & 45.7 & 39.1 & & \\
\hline & 90 & 52.0 & 44.8 & 49.6 & 42.4 & & & 48.4 & 46.0 & & & 50.8 & 43.6 & & \\
\hline & 120 & 55.9 & 50.9 & 54.6 & 48.1 & & & 53.4 & 51.4 & & & 55.3 & 49.5 & & \\
\hline & 0 & 44.5 & 43.7 & 45.4 & 43.9 & \multirow{5}{*}{2.7} & & 44.1 & 44.6 & \multirow{5}{*}{2.1} & & 44.9 & 43.8 & \multirow{5}{*}{2.1} & \\
\hline & 30 & 31.4 & 28.6 & 29.7 & 31.3 & & & 30.0 & 30.5 & & & 30.5 & 30.0 & & \\
\hline & 60 & 35.2 & 33.7 & 34.7 & 33.0 & & & 34.4 & 33.9 & & & 35.0 & 33.3 & & \\
\hline & 90 & 40.9 & 37.5 & 39.1 & 36.5 & & & 39.2 & 37.8 & & & 40.0 & 37.0 & & \\
\hline & 120 & 44.3 & 41.9 & 43.2 & 38.3 & & & 43.1 & 40.8 & & & 43.7 & 40.1 & & \\
\hline
\end{tabular}

${ }^{1} n=97$ for adults; ${ }^{2} n=82$ for elderly; ${ }^{3}$ determined by ANOVA between age groups, differences are statistically significant when $p<0.05$. Mean data are presented for drink type (NPWL, NPMT, HPWL, HPMT), drink composition (NP, HP) and drink quantity (WL, MT); HP: high protein, HPMT: high-protein maintenance, HPWL: high-protein weight loss, MT: weight maintenance, NP: normal protein, NPMT: normal-protein maintenance, NPWL: normal-protein weight loss, VAS: visual analog scale, WL: weight loss. 


\subsection{Test Drink Pleasantness and Satisfaction}

There was a significant difference in pleasantness ratings between drinks for the children and adolescents and the adults and elderly, with a significantly higher rating for the NPWL drink, in comparison to the NPMT, HPWL and HPMT drinks (children and adolescents $(n=212): 5.14,4.80$, 4.97, 4.60, respectively; $p<0.001$; SED: 0.12; adults and elderly $(n=179): 68.4,64.6,64.7,62.5 \mathrm{~mm}$, respectively; $p=0.002$; SED: 1.9). Participants also preferred the NP over HP composition (children and adolescents: 4.97, 4.78, respectively; $p=0.032$; SED: 0.09; adults and elderly: 66.5, $63.6 \mathrm{~mm}$, respectively; $p=0.012$; SED: 1.3 ) and the WL over the MT quantity (children and adolescents: $5.05,4.70$, respectively; $p<0.001$; SED: 0.09; adults and elderly: 66.5, $63.6 \mathrm{~mm}$, respectively; $p=0.04$; SED: 1.3 ). Average pleasantness ratings were significantly higher in the ABDN children and adolescent cohort $(n=84)$ compared to the HUA cohort $(n=128 ; 5.86,3.89$, respectively; $p<0.001$; SED: 0.22$)$.

Average satisfaction ratings were significantly higher in the children $(n=103)$ compared to the adolescents $(n=109 ; 5.17,4.27$, respectively; $p=0.009$; SED: 0.20$)$. Children and adolescent females $(n=107)$ reported significantly higher average satisfaction ratings compared to males $(n=105 ; 4.95$, 4.49 , respectively; $p=0.037$; SED: 0.20 ), and average satisfaction ratings were higher in the ABDN children and adolescent cohort $(n=84)$ compared to the HUA cohort $(n=128 ; 5.45,3.99$, respectively; $p<0.001$; SED: 0.20). There were no differences between age groups, weight categories, genders or sites and no effect of drink on satisfaction ratings in the adults and elderly ( $n=179$; data not shown).

\subsection{Food Reward: $L F P Q$}

Fat bias scores (liking and wanting scores for high-fat relative to low-fat foods) and sweet bias scores (scores for sweet relative to savory foods) were compared according to age, BMI, gender and site (Table S6). There was a main effect of age group on liking $(p=0.001)$ and wanting $(p<0.001)$ for high-fat food. Post hoc analyses showed that the elderly had the lowest fat preference, followed by adults, and that both groups showed a clear preference (liking and wanting) for low-fat relative to high-fat foods. Adolescents showed a greater liking and wanting for high-fat relative to low-fat food. There was also an effect of age group on wanting for sweet foods $(p<0.001)$, with a greater wanting for sweet in children, adolescents and elderly compared to adults $(p<0.05)$. For BMI, while there were no group differences for liking and wanting fat bias, liking $(p=0.047)$ and wanting $(p=0.059)$ sweet bias tended to be greater in normal weight than overweight participants. There was no main effect of gender. There was a main effect of site on liking and wanting for sweet (both $p=0.019$ ) and high-fat (both $p<0.001$ ) foods, with the ABDN population showing a greater sweet and fat preference compared to HUA.

\subsection{Biomarkers}

Note that, of the 179 adult and elderly participants, four normal weight adults and three normal weight elderly from ABDN were unable to provide blood samples, therefore, 172 participants were included in the biomarker analyses.

\subsubsection{Appetite Hormones}

Differences in GLP-1, PYY and ghrelin concentrations between age groups, weight categories, genders and sites in response to all test drinks combined are presented in Figure 2 (GLP-1), Figure 3 (PYY) and Figure 4 (ghrelin). GLP-1 and PYY baseline concentrations did not differ significantly between groups for age, BMI, gender or site comparisons. Ghrelin baseline concentrations did not differ significantly between age groups, but baseline differences are reported for gender, $\mathrm{BMI}$ and site comparisons (all $p<0.001$ ). Plasma concentrations of GLP-1 (Figure 2A) and PYY (Figure 3A) were significantly greater in the elderly than the adults (both $p<0.001$ ), however, there were no significant differences in ghrelin release between age groups (Figure $4 \mathrm{~A} ; p=0.119$ ). There were no significant differences in GLP-1 (Figure 2B; $p=0.996$ ) or PYY (Figure 3B; $p=0.826$ ) responses between normal 
weight and overweight participants, however, normal weight participants exhibited significantly greater ghrelin concentrations when compared to overweight participants (Figure 4B; $p<0.001$ ). Concentrations of all three hormones were greater in females in comparison to males $(p=0.039$, $p=0.028$ and $p<0.001$ for GLP-1 (Figure 2C), PYY (Figure 3C) and ghrelin (Figure 4C), respectively). There were no differences in GLP-1 (Figure 2D) or PYY (Figure 3D) concentrations between ABDN and HUA participants, though ghrelin concentrations were greater in the ABDN cohort compared to the HUA cohort (Figure 4D; $p<0.001$ ). Interestingly, ghrelin differences between sites could not be explained by differences in body composition or gender.

Table 8 presents the gut hormones (GLP-1, PYY and ghrelin) drink type, composition and quantity $x$ time interactions for all participants combined. There was a significant effect of drink type on concentrations of GLP-1 and PYY (both $p<0.001)$ and ghrelin $(p<0.005)$. The HP test drinks elicited a significantly greater increase in GLP-1 and PYY (both $p<0.001$ ) in comparison to the NP drinks, however, protein content did not significantly affect ghrelin $(p=0.710)$. The MT test drinks elicited a significantly greater increase in GLP-1 and PYY (both $p<0.001$ ) in comparison to the WL drinks (both $p<0.001$ ), while ghrelin was suppressed to a significantly greater extent in response to the MT drinks compared to the WL drinks $(p<0.001)$.

Pooled data from adults and elderly demonstrated that GLP-1 and PYY concentrations were negatively associated with ad libitum EI (both $p<0.001$ ), while there was no significant association between ghrelin and ad libitum EI $(p=0.770)$. PYY concentrations were negatively associated with composite appetite score $(p=0.028)$, while the association between GLP-1 concentrations and composite appetite score approached significance $(p=0.052)$. There was no significant association between ghrelin concentrations and composite appetite score $(p=0.605)$.

A

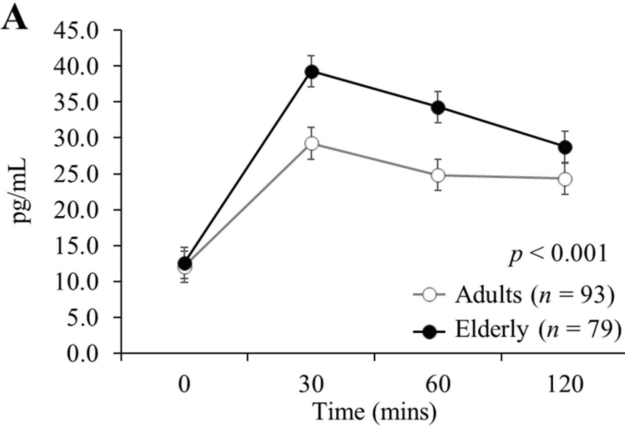

C

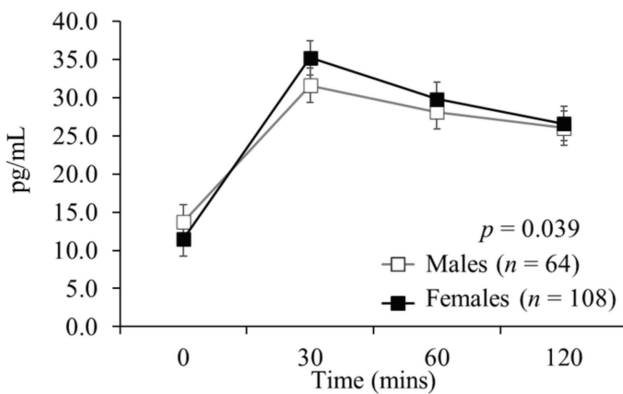

B

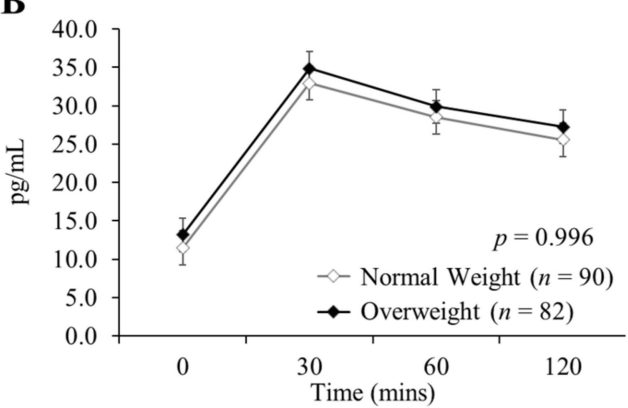

D

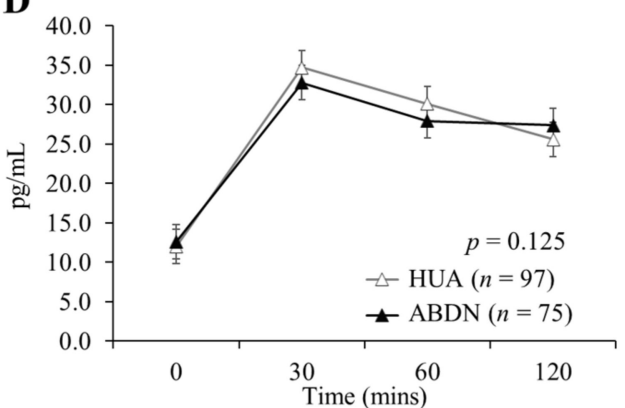

Figure 2. Plasma concentrations of GLP-1 in the adult and elderly cohorts in response to all test drinks combined. Data are presented as mean \pm SED, $n=172$; seven ABDN participants (four normal weight adults, three normal weight elderly) did not complete this measurement. Determined using an electrochemical luminescence immunoassay kit, values were analyzed as repeated measurements using ANOVA, differences are statistically significant when $p<0.05$. (A) Comparison of age group, (B) comparison of BMI group, (C) comparison of gender, (D) comparison of site. ABDN: Scotland, GLP-1: glucagon-like peptide 1, HUA: Greece. 
A

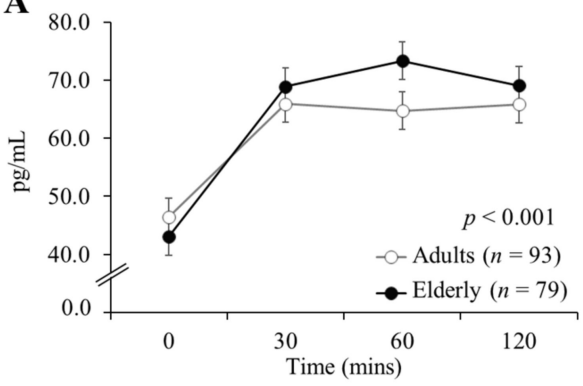

C

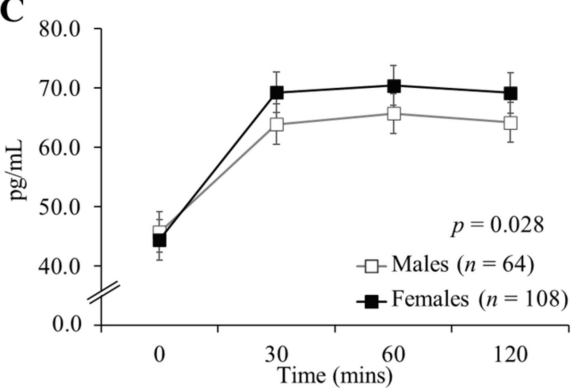

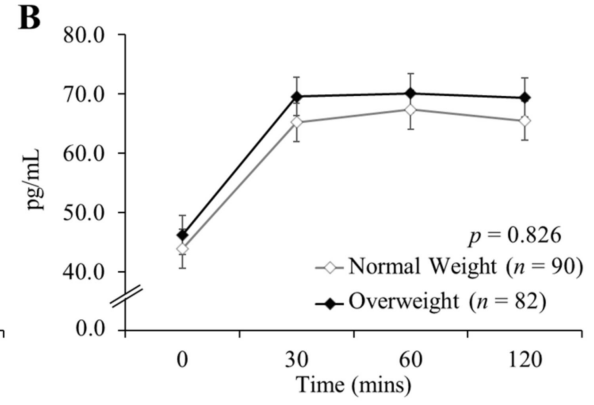

D

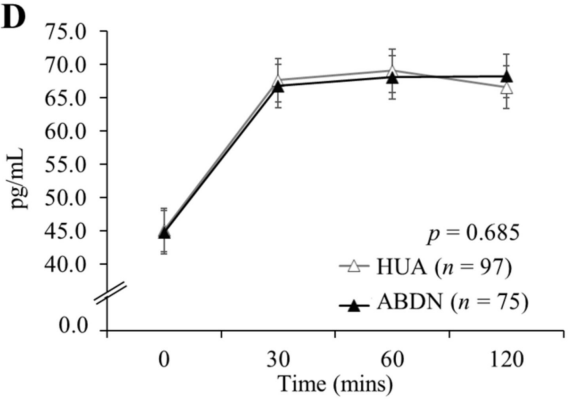

Figure 3. Plasma concentrations of PYY in the adult and elderly cohorts in response to all test drinks combined. Data are presented as mean $\pm \mathrm{SED}, n=172$; seven $\mathrm{ABDN}$ participants (four normal weight adults, three normal weight elderly) did not complete this measurement. Determined using an electrochemical luminescence immunoassay kit, values were analyzed as repeated measurements using ANOVA, differences are statistically significant when $p<0.05$. (A) Comparison of age group, (B) comparison of BMI group, (C) comparison of gender, (D) comparison of site. ABDN: Scotland, HUA: Greece, PYY: peptide YY.

A

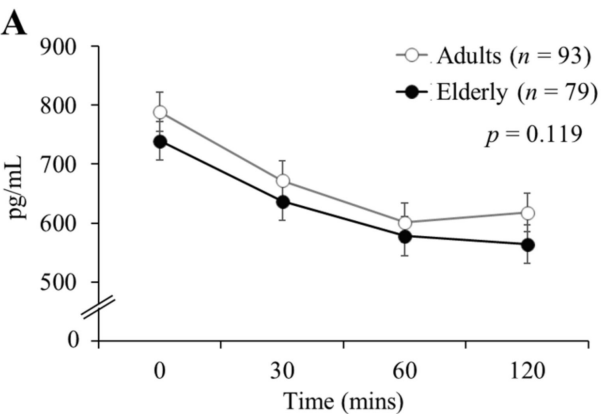

C

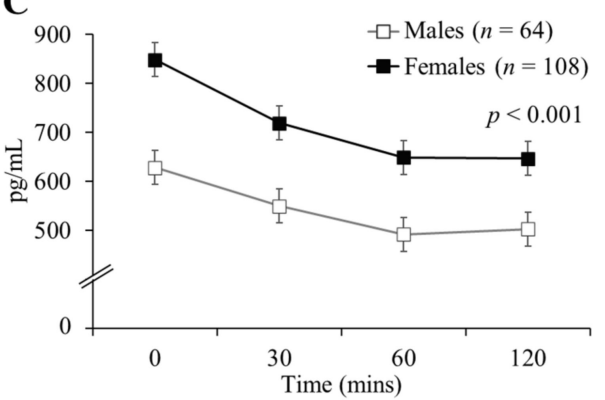

B

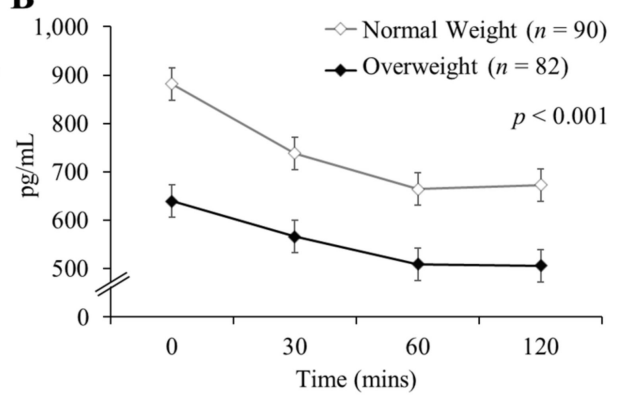

D

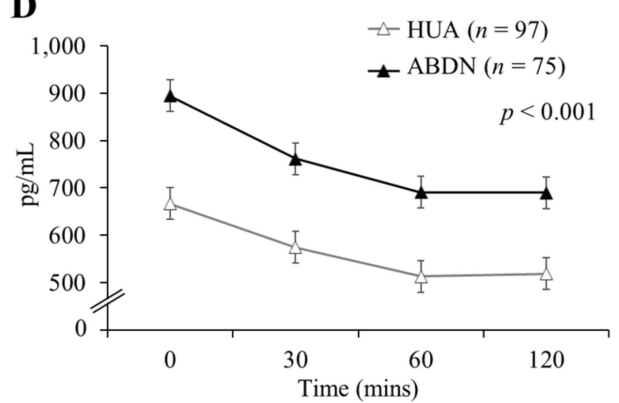

Figure 4. Plasma concentrations of ghrelin in the adult and elderly cohorts in response to all test drinks combined. Data are presented as mean \pm SED, $n=172$; seven ABDN participants (four normal weight adults, three normal weight elderly) did not complete this measurement. Determined using a human-specific radioimmunoassay kit, values were analyzed as repeated measurements using ANOVA, differences are statistically significant when $p<0.05$. (A) Comparison of age group, (B) comparison of BMI group, (C) comparison of gender, (D) comparison of site. ABDN: Scotland, HUA: Greece. 
Table 8. Combined adult and elderly ${ }^{1}$ appetite hormone concentrations at baseline and in response to test drink type, composition and quantity.

\begin{tabular}{|c|c|c|c|c|c|c|c|c|c|c|}
\hline Drink & Time (min) & GLP-1 (pg/mL) & SED & $\begin{array}{c}\text { Drink.Time } \\
\text { Interaction, } p^{2}\end{array}$ & PYY (pg/mL) & SED & $\begin{array}{c}\text { Drink.Time } \\
\text { Interaction, } p^{2}\end{array}$ & Ghrelin (pg/mL) & SED & $\begin{array}{c}\text { Drink.Time } \\
\text { Interaction, } p^{2}\end{array}$ \\
\hline \multirow{4}{*}{ NPWL } & 0 & 12.7 & \multirow{15}{*}{1.3} & \multirow{15}{*}{$<0.001$} & 46.1 & \multirow{15}{*}{1.9} & \multirow{15}{*}{$<0.001$} & 773 & \multirow{15}{*}{12} & \multirow{15}{*}{$<0.005$} \\
\hline & 30 & 33.1 & & & 68.3 & & & 651 & & \\
\hline & 60 & 26.6 & & & 69.6 & & & 596 & & \\
\hline & 120 & 21.8 & & & 65.2 & & & 604 & & \\
\hline \multirow{4}{*}{ NPMT } & 0 & 11.9 & & & 43.5 & & & 762 & & \\
\hline & 30 & 37.1 & & & 71.3 & & & 659 & & \\
\hline & 60 & 31.3 & & & 74.3 & & & 584 & & \\
\hline & 120 & 28.7 & & & 73.4 & & & 574 & & \\
\hline \multirow{3}{*}{ HPWL } & 30 & 31.3 & & & 63.2 & & & 661 & & \\
\hline & 60 & 27.6 & & & 63.4 & & & 603 & & \\
\hline & 120 & 26.3 & & & 64.2 & & & 622 & & \\
\hline \multirow{4}{*}{ HPMT } & 0 & 12.5 & & & 45.2 & & & 763 & & \\
\hline & 30 & 33.9 & & & 66.4 & & & 653 & & \\
\hline & 60 & 31.1 & & & 67.3 & & & 578 & & \\
\hline & 120 & 28.7 & & & 66.5 & & & 572 & & \\
\hline \multirow{3}{*}{ NP } & 0 & 12.3 & \multirow{6}{*}{1.1} & \multirow{6}{*}{$<0.001$} & 44.8 & \multirow{6}{*}{1.5} & \multirow{6}{*}{$<0.001$} & 767 & & \\
\hline & 30 & 35.1 & & & 69.8 & & & 655 & & \\
\hline & 60 & 29.0 & & & 72.0 & & & 590 & & \\
\hline \multirow{3}{*}{$\mathrm{HP}$} & 30 & 32.6 & & & 64.8 & & & 657 & & \\
\hline & 60 & 29.4 & & & 65.4 & & & 591 & & \\
\hline & 120 & 27.5 & & & 65.3 & & & 597 & & \\
\hline \multirow{4}{*}{ WL } & 0 & 12.4 & \multirow{8}{*}{1.1} & \multirow{8}{*}{$<0.001$} & 45.5 & & & 770 & & \\
\hline & 30 & 32.2 & & & 65.7 & & & 656 & & \\
\hline & 60 & 27.1 & & & 66.5 & & & 599 & & \\
\hline & 120 & 24.1 & & & 64.7 & & & 613 & & \\
\hline \multirow{4}{*}{ MT } & 0 & 12.2 & & & 44.4 & 1.5 & $<0.001$ & 763 & 9 & $<0.001$ \\
\hline & 30 & 35.5 & & & 68.8 & & & 656 & & \\
\hline & 60 & 31.2 & & & 70.8 & & & 581 & & \\
\hline & 120 & 28.7 & & & 69.9 & & & 573 & & \\
\hline
\end{tabular}

${ }^{1} n=172$; seven ABDN participants (four normal weight adults, four normal weight elderly) did not complete this measurement; ${ }^{2}$ determined by ANOVA, differences are statistically significant when $p<0.05$. Mean data are presented for drink type (NPWL, NPMT, HPWL, HPMT), drink composition (NP, HP) and drink quantity (WL, MT); GLP-1: glucagon-like peptide 1, HP: high protein, HPMT: high-protein maintenance, HPWL: high-protein weight loss, MT: weight maintenance, NP: normal protein, NPMT: normal-protein maintenance,

NPWL: normal-protein weight loss, PYY: pancreatic peptide YY, WL: weight loss. 
There were no significant differences in fasting leptin concentrations between adult and elderly participants (adults: $18.21 \mathrm{ng} / \mathrm{mL}$; elderly: $20.86 \mathrm{ng} / \mathrm{mL} ; p=0.408$; SED: 2.08). It is well established that obesity enhances the synthesis and release of leptin and, as anticipated, leptin concentrations were significantly higher in overweight participants compared to normal weight participants (overweight: $29.49 \mathrm{ng} / \mathrm{mL}$; normal weight: $10.26 \mathrm{ng} / \mathrm{mL} ; p<0.001$; SED: 2.04$)$. Females exhibited significantly greater concentrations of leptin than males (females: $26.55 \mathrm{ng} / \mathrm{mL}$; males: $7.40 \mathrm{ng} / \mathrm{mL} ; p<0.001$; SED: 2.12 ). Leptin concentrations were also significantly greater in HUA participants compared to the ABDN cohort (HUA: $21.36 \mathrm{ng} / \mathrm{mL}$; ABDN: $16.93 \mathrm{ng} / \mathrm{mL} ; p=0.016$; SED: 1.98). The gender and site differences can be explained by differences in body composition.

\subsubsection{Glucose Homeostasis}

Table S7 presents the group x time interactions for glucose and insulin concentrations, and includes HOMA-IR, IGI and IGR. Elderly participants exhibited significantly greater concentrations of glucose $(p<0.001)$, insulin $(p<0.001)$ and HOMA-IR $(p<0.001)$ compared to adults. As expected, glucose homeostasis was significantly influenced by BMI, with glucose $(p<0.001)$, insulin $(p<0.001)$, HOMA-IR $(p<0.001)$ and IGR $(p=0.006)$ greater in overweight compared to normal weight participants. Glucose $(p=0.036)$ and IGR $(p=0.005)$ were significantly greater in females compared to males. Glucose $(p=0.008)$, insulin $(p=0.005)$, HOMA-IR $(p=0.017)$ and IGR $(p=0.005)$ were significantly greater in the HUA cohort compared to the ABDN cohort. These significant group $\times$ time interactions were due to delayed insulin responses in elderly, overweight and HUA participants. Furthermore, differences in insulin concentrations between sites can be explained by differences in body composition.

There were significant drink type $(p<0.001)$, composition $(p<0.05)$ and quantity $(p<0.001) \times$ time interaction effects for glucose, insulin, HOMA-IR, IGI and IGR (Table S8).

\section{Discussion}

In relation to the primary aim of this study, the current novel findings demonstrate that ad libitum EI did not differ significantly between age groups, BMIs, genders or geographical locations, though composite appetite score was lower in the elderly subjects compared to the younger adults in response to drink type and quantity. The elderly group exhibited greater postprandial levels of GLP-1 and PYY than the adult group, but ghrelin release was not affected by age. Concentrations of all appetite hormones were greater in females compared to males, while ghrelin levels were lower and fasting leptin levels were higher in overweight compared to normal weight participants. Furthermore, elevated ghrelin release and suppressed fasting leptin levels were observed in the ABDN cohort in comparison to the HUA cohort. As regards the secondary aim, ad libitum EI was not affected by drink composition, though concentrations of GLP-1 and PYY were higher in response to the HP compared to the NP test drinks. In addition, as might be expected, in response to the WL drink quantity, ad libitum EI was elevated, GLP-1 and PYY levels were lower and ghrelin concentrations were higher in comparison to the MT drink quantity.

\subsection{Ad Libitum EI and Subjective Appetite}

In the present study, there were no significant effects of the test meal on ad libitum EI between weight categories, gender, site or age; albeit we noted a trend towards differences between age groups in response to the quantity of drink provided below or at maintenance requirements. This approached significance, in part explained by the lower intakes in the elderly participants, which was also detected in their significantly lower subjective appetite score. Other authors have highlighted differences in appetite suppression between young and older healthy participants in response to protein and energy load [44], and this warrants further investigation to explore the influence of ageing on mechanisms of protein-induced satiety. Although we assessed subsequent ad libitum EI $2 \mathrm{~h}$ after the breakfast drink, we did not measure $24 \mathrm{~h} \mathrm{EI}$, so it may be that energy compensation occurred later in the day. Belza et al. [25] also examined the effects of consuming an NP vs. HP test drink in adults. The HP drink 
led to reduced hunger and increased satiety compared to the NP drink. This might be because the HP drink that Belza et al. [25] used provided 50\% energy from protein or $88.4 \mathrm{~g}$ protein per dose; there may be a threshold absolute concentration of protein required to stimulate protein-induced satiety, and the amount of protein supplied in our HP drink (10 g) may have fallen below this threshold. Furthermore, food form could be important for appetite control across the lifecourse. Indeed, Leidy et al. [23] demonstrated that a solid meal reduced lunch intake by approximately $480 \mathrm{~kJ}$ compared to a liquid meal in adolescents. By the nature of our current study design, we did not investigate the form of protein delivery and it is unclear as to whether a solid version would elicit greater changes.

To the authors' knowledge, this is one of the few studies to report the effects of study visit number on ad libitum EI. Interestingly, we observed that the children's ad libitum EI was lower on the final visit compared to the first visit, in agreement with previous research [45], while the adult and elderly ad libitum EI was higher. Possible explanations for these findings may include children habituating to the buffet items following their initial, novel exposure to the buffet during visit 1 , and the older age groups initially experiencing heightened feelings of anxiety before acclimatizing to the environment, as demonstrated previously [46]. Future studies may consider incorporating a familiarization/acclimatization session when assessing ad libitum EI, to reduce the effects of study visit order.

\subsection{Food Reward: LFPQ}

A greater liking and wanting for low-fat relative to high-fat foods and non-sweet relative to sweet foods was shown in the Greek participants. This difference may reflect in part the cultural norms for consuming sweet foods in the morning in Scotland but may also be due to the greater availability of fresh fruit and vegetables and the more traditional rather than "'westernized" diet in Greece $[47,48]$. Indeed, a north-south European differentiation in food habits consistent with these findings has previously been proposed [49]. We also found an age effect, with adults and the elderly having a greater liking and wanting for low-fat food compared to children and adolescents, and adults having a lower wanting of sweet foods compared to similarly high sweet wanting scores in children, adolescents and the elderly. Very few studies have examined food preferences across the lifecourse and, to our knowledge, no studies have examined both dimensions of fat and sweet taste in food. The findings on sweet taste preference are consistent with one psychophysical study showing that optimally preferred sucrose concentrations were higher for the elderly than for other age groups, except for the children [50]. As regards fat preferences, it is noted that the ability to accurately assess the fat content of foods is limited in humans, but adults may be more responsive to visual cues indicating the healthiness of food, which could influence food choice [51].

\subsection{Biomarkers}

GLP-1 is co-secreted with PYY by L cells in the lower intestine, with concentrations of both hormones increasing in response to a meal and inducing acute satiety [52-54]. Deficiencies in GLP-1 and PYY have been reported in obese individuals [55-58], although not consistently [59-61] and not in the present study. We do, however, report higher postprandial concentrations of GLP-1 and PYY in the elderly and in females, which in the long term could partially facilitate weight reduction. Ageing modifies the gastrointestinal tract, causing alterations in gut hormone secretion and feedback mechanisms, which slow gastric emptying [62]. Furthermore, authors have observed slower gastric emptying rates in females compared to males $[63,64]$. Elevated concentrations of GLP-1 and PYY contribute to delayed gastric emptying and prolonged satiety [56,65]. Therefore, we speculate that slower gastric emptying in the elderly and female groups may have partially accounted for elevated postprandial levels of GLP-1 and PYY. In addition, gastric emptying and satiety hormones have been shown to fluctuate depending on the phase of the menstrual cycle [66-68], highlighting the important role of sex hormones in appetite control. 
Ghrelin is the only known gastrointestinal hormone to increase food intake [69]. Its concentrations peak prior to meal initiation and are suppressed by nutrient intake [69]. We report elevated fasting ghrelin concentrations in the normal weight compared to overweight participants and in females vs. males, as shown previously [70], however, postprandial patterns of response were similar. Ghrelin concentrations did not differ between adults and elderly, which agrees with previous data [71-75], though findings to the contrary have also been reported [76-79]. Ghrelin exists as two isoforms: acyl ghrelin, which stimulates energy intake [80,81] and des-acyl ghrelin, which may act independently from acyl ghrelin [82]. Most studies, including the present study, reporting no differences in ghrelin concentrations between younger and older adults [71-73,75], have measured total ghrelin (acyl and des-acyl ghrelin) only. However, studies measuring acyl ghrelin observe lower concentrations and an impaired postprandial response in the elderly [77-79]. Therefore, the form of ghrelin measured may contribute to discrepancies in the literature and merits further investigation.

Although insulin is predominantly considered a principal regulator of glucose metabolism, it also acts on the arcuate nucleus of the hypothalamus to signal satiety [83]. Studies demonstrating increased satiety with age also report greater postprandial insulin concentrations in older compared to younger adults $[73,78,84]$, in agreement with our findings. Insulin modulates changes in the circulation of leptin [85] and ghrelin [86] and may induce satiety indirectly by amplifying the anorectic actions of leptin and/or suppressing ghrelin secretion [86,87]. In the present study, we did not observe differences in leptin or ghrelin concentrations between adults and the elderly, suggesting that greater insulin secretion in the elderly was not sufficient to cause age-related differences in leptin and ghrelin release.

This is one of the few studies to compare appetite control in different geographical locations. We observed lower ghrelin concentrations in HUA participants compared to those in ABDN. However, differences at baseline were accountable for postprandial differences between each "site". Interestingly, we also observed elevated fasting leptin concentrations and postprandial insulin concentrations in the HUA cohort compared to the ABDN cohort, which may have partially modulated ghrelin expression. These are novel findings and not likely due to technical issues since processing, storage and analysis were identical, instead, differences in leptin and insulin levels appear to be associated with differences in body composition between the two locations, though body composition did not account for differences in ghrelin concentrations and neither did gender. Future studies may consider the influence of geographical location on variations in appetite control, as other authors have suggested that differences are not related to habitual diet [88].

In the current study, we observed a significant decrease in ghrelin concentrations in response to the caloric load, but not the protein amount. This lack of protein-induced dose-dependent effect is reported by other authors $[25,89]$. It has been suggest that the postprandial decrease in ghrelin may be mediated through stimulation of gastric inhibitory polypeptide (GIP) and glucagon $[25,90,91]$, possibly linked to gastric emptying, but also the carbohydrate content of the meal $[25,91]$. We also suggest that interactions between the protein and carbohydrate content is likely to influence ghrelin release, and that dietary carbohydrate may be a more potent stimulator than protein. Interestingly, in the current study, the HP drinks increased GLP-1 and PYY in comparison to the NP drinks. Belza et al. [25] provide a concise commentary on this aspect and suggest that these two hormones, in combination, do affect appetite after a protein-rich meal.

\subsection{Strengths and Limitations}

The present study has several strengths, which include having a controlled diet intervention study conducted as a randomized crossover design in a large cohort taking account of age, body size, gender and geographical location. For some factors (such as drink), this study has the strengths of a crossover design, whereas for others (such as age), there are the unavoidable limitations of studying observable factors. As with any lab-based dietary intervention study, there are limitations, such as limited ecological validity, the amount and type of protein and many phenotypic effects which have not been investigated. The iso-energetic load for the meals was achieved by reducing carbohydrate 
content, so we cannot rule out the effect of this lower carbohydrate nutrient profile of the high-protein meal to contribute to the study results. We presented unadjusted $p$-values for comparing treatment groups in tests of several variables, to preserve the power of the study, so although there are clear patterns of significant differences, there is a risk that a small proportion of these are type I errors. Therefore, significant $p$-values presented within the present study come with this caveat, which should be considered when interpreting our findings. We recruited people motivated to respond to a diet trial and, consequently, this is not a truly random sample. Furthermore, had we recruited a much larger sample size, this may have allowed the statistical results to be generalized to a larger population or phenotype. Finally, long-term intervention and monitoring across the lifecourse to assess the mechanisms underlying changes in appetite control were out with the scope of this study.

\section{Conclusions}

The primary aim of this study was to describe the acute regulation of appetite across the lifecourse, thus being able to detect differences between four different age groups (children, adolescents, adult and elderly), two different weight categories (normal weight and overweight), the two genders (male and female) and two European sites (Aberdeen, Scotland and Athens, Greece). The present study shows that the elderly reported lower subjective appetite ratings in response to the different drink types and quantities in comparison to the adults. Furthermore, in agreement with Di Francesco et al. [73], postprandial anorexigenic signals prevailed over orexigenic signals in the elderly, which over time could induce an energy deficit and accentuate the anorexia of ageing. In addition to age, differences in appetite hormone concentrations between BMIs, genders and geographical locations were also observed, the latter of which being of particular interest, as location is rarely considered in the context of acute appetite control. Future research may consider expanding upon our findings and examining the role of appetitive neuronal circuits in food-gut-brain interactions across the lifecourse.

Supplementary Materials: The following are available online at http://www.mdpi.com/2072-6643/12/12/3710/s1, Table S1: Schofield equations for estimating BMR, Table S2: Nutritional values of the foods and drinks served at the ad libitum buffet-style test meal, Table S3: Number of participants completed within each group at both sites, Table S4: Buffet-style test meal ad libitum energy and macronutrient intake $(n=391)$, Table S5: Effect of visit number on ad libitum EI, Table S6: Mean food reward (liking and wanting) results, Table S7: Combined adult and elderly glucose homeostasis, by group, at baseline and post-test drink consumption, Table S8: Combined adult and elderly glucose homeostasis at baseline and in response to test drink type, composition and quantity, Figure S1: CONSORT diagram summarizing participant flow. The number of participants from both study sites who were recruited, enrolled, allocated to intervention, discontinued, included in the analyses and completed are presented. ABDN: Scotland, HUA: Greece.

Author Contributions: Y.M., J.G.M. and A.M.J. conceptualization; D.R.C., W.B., C.L.F., O.A. and A.G. data curation; G.W.H., G.F. and K.B. formal analysis; Y.M., J.G.M. and A.M.J. funding acquisition; D.R.C., W.B., C.L.F., O.A., A.G., C.L.M., J.J.H. and A.M.J. investigation; D.R.C., W.B., O.A., G.F., C.L.M., J.J.H., K.V.N. and A.M.J. methodology; G.W.H. project administration; G.F., C.L.M., J.J.H. and K.V.N. resources; Y.M. and J.G.M. supervision; D.R.C., W.B., C.L.F., G.F. and A.M.J. writing—original draft; G.W.H., Y.M., O.A., A.G., K.B., C.L.M., J.J.H., K.V.N. and J.G.M. writing-review \& editing. All authors have read and agreed to the published version of the manuscript.

Funding: This research was funded by the European Union's Seventh Framework Programme FP7-KBBE-2010-4 under grant agreement No: 266408. Authors from the University of Aberdeen, Rowett Institute gratefully acknowledge financial support from the Scottish Government as part of the RESAS Strategic Research Programme at the Rowett Institute.

Acknowledgments: We thank Sylvia Stephen, Karen Taylor, Ruth Melican, Rachel Malone, Tara Lyons, Argyri Tsipra, Charlotte Zeller, Miroslaw Kasprzak, Niamh Maloney, Evie Nikokavoura, Laura Bardon, Zahra Mansy and the staff at the Human Nutrition Unit for their assistance with the dietary preparation and participant data collection. The authors would also like to thank all the participants for their time and effort.

Conflicts of Interest: Dr Klaske van Norren worked as a Wageningen University consultant for Nutricia Research in Utrecht, a medical Nutrition company. No other author has a conflict of interest to declare. The founding sponsors had no role in the design of the study; in the collection, analyses, or interpretation of data; in the writing of the manuscript, and in the decision to publish the results. 


\section{References}

1. World Health Organization. Diet, Nutrition and the Prevention of Chronic Diseases. Report of a Joint WHO/FAO Expert Consultation; WHO Technical Report Series No. 916; World Health Organization: Geneva, Switzerland, 2003.

2. Darnton-Hill, I.; Nishida, C.; James, W.P.T. A life course approach to diet, nutrition and the prevention of chronic diseases. Public Health Nutr. 2004, 7, 101-121. [CrossRef] [PubMed]

3. Donaldson, A.I.C.; Johnstone, A.M.; de Roos, B.; Myint, P.K. Role of protein in healthy ageing. Eur. J. Integr. Med. 2018, 23, 32-36. [CrossRef]

4. Zac-Varghese, S.; Tan, T.; Bloom, S.R. Hormonal interactions between gut and brain. Discov. Med. 2010, 10, 543-552. [PubMed]

5. Bilman, E.; van Kleef, E.; van Trijp, H. External cues challenging the internal appetite control system-Overview and practical implications. Crit. Rev. Food Sci. Nutr. 2017, 57, 2825-2834. [CrossRef] [PubMed]

6. Murphy, K.G.; Bloom, S.R. Gut hormones in the control of appetite. Exp. Physiol. 2004, 89, 507-516. [CrossRef]

7. Simpson, K.A.; Bloom, S.R. Appetite and Hedonism: Gut Hormones and the Brain. Endocrinol. Metab. Clin. 2010, 39, 729-743. [CrossRef] [PubMed]

8. Juonala, M.; Magnussen, C.G.; Berenson, G.S.; Venn, A.; Burns, T.L.; Sabin, M.A.; Srinivasan, S.R.; Daniels, S.R.; Davis, P.H.; Chen, W.; et al. Childhood Adiposity, Adult Adiposity, and Cardiovascular Risk Factors. N. Engl. J. Med. 2011, 365, 1876-1885. [CrossRef]

9. Rendina, D.; Campanozzi, A.; De Filippo, G. Methodological approach to the assessment of the obesogenic environment in children and adolescents: A review of the literature. Nutr. Metab. Cardiovasc. Dis. 2019, 29, 561-571. [CrossRef]

10. Marschner, A.; Mell, T.; Wartenburger, I.; Villringer, A.; Reischies, F.M.; Heekeren, H.R. Reward-based decision-making and aging. Brain Res. Bull. 2005, 67, 382-390. [CrossRef]

11. Dreher, J.-C.; Meyer-Lindenberg, A.; Kohn, P.; Berman, K.F. Age-related changes in midbrain dopaminergic regulation of the human reward system. Proc. Natl. Acad. Sci. USA 2008, 105, 15106-15111. [CrossRef]

12. Pelchat, M.L. Food Cravings in Young and Elderly Adults. Appetite 1997, 28, 103-113. [CrossRef] [PubMed]

13. Koehler, K.M. The New Mexico Aging Process Study. Nutr. Rev. 1994, 52, S34-S37. [CrossRef] [PubMed]

14. Zhu, K.; Devine, A.; Suleska, A.; Tan, C.Y.; Toh, C.Z.J.; Kerr, D.; Prince, R.L. Adequacy and change in nutrient and food intakes with aging in a seven-year cohort study in elderly women. J. Nutr. Health Aging 2010, 14, 723-729. [CrossRef]

15. Morley, J.E. Anorexia of aging: Physiologic and pathologic. Am. J. Clin. Nutr. 1997, 66, 760-773. [CrossRef] [PubMed]

16. Briefel, R.R.; McDowell, M.A.; Alaimo, K.; Caughman, C.R.; Bischof, A.L.; Carroll, M.D.; Johnson, C.L. Total energy intake of the US population: The third National Health and Nutrition Examination Survey, 1988-1991. Am. J. Clin. Nutr. 1995, 62, 1072S-1080S. [CrossRef] [PubMed]

17. Mercer, J.G.; Johnstone, A.M.; Halford, J.C.G. Approaches to influencing food choice across the age groups: From children to the elderly. Proc. Nutr. Soc. 2015, 74, 149-157. [CrossRef]

18. Nickols-Richardson, S.M.; Coleman, M.D.; Volpe, J.J.; Hosig, K.W. Perceived Hunger Is Lower and Weight Loss Is Greater in Overweight Premenopausal Women Consuming a Low-Carbohydrate/High-Protein vs High-Carbohydrate/Low-Fat Diet. J. Am. Diet. Assoc. 2005, 105, 1433-1437. [CrossRef]

19. Lejeune, M.P.; Westerterp, K.R.; Adam, T.C.; Luscombe-Marsh, N.D.; Westerterp-Plantenga, M.S. Ghrelin and glucagon-like peptide 1 concentrations, 24-h satiety, and energy and substrate metabolism during a high-protein diet and measured in a respiration chamber. Am. J. Clin. Nutr. 2006, 83, 89-94. [CrossRef]

20. Johnstone, A.M.; Horgan, G.W.; Murison, S.D.; Bremner, D.M.; Lobley, G.E. Effects of a high-protein ketogenic diet on hunger, appetite, and weight loss in obese men feeding ad libitum. Am. J. Clin. Nutr. 2008, 87, 44-55. [CrossRef]

21. Wolfe, R.R. The role of dietary protein in optimizing muscle mass, function and health outcomes in older individuals. Br. J. Nutr. 2012, 108 (Suppl. 2), S88-S93. [CrossRef]

22. Veldhorst, M.; Smeets, A.; Soenen, S.; Hochstenbach-Waelen, A.; Hursel, R.; Diepvens, K.; Lejeune, M.; Luscombe-Marsh, N.; Westerterp-Plantenga, M. Protein-induced satiety: Effects and mechanisms of different proteins. Physiol. Behav. 2008, 94, 300-307. [CrossRef] [PubMed] 
23. Leidy, H.J.; Bales-Voelker, L.I.; Harris, C.T. A protein-rich beverage consumed as a breakfast meal leads to weaker appetitive and dietary responses v. a protein-rich solid breakfast meal in adolescents. Br. J. Nutr. 2011, 106, 37-41. [CrossRef] [PubMed]

24. Nguo, K.; Bonham, M.; Truby, H.; Barber, E.; Brown, J.; Huggins, C. Effect of Macronutrient Composition on Appetite Hormone Responses in Adolescents with Obesity. Nutrients 2019, 11, 340. [CrossRef] [PubMed]

25. Belza, A.; Ritz, C.; Sørensen, M.Q.; Holst, J.J.; Rehfeld, J.F.; Astrup, A. Contribution of gastroenteropancreatic appetite hormones to protein-induced satiety. Am. J. Clin. Nutr. 2013, 97, 980-989. [CrossRef] [PubMed]

26. Association, W.M. World Medical Association Declaration of Helsinki: Ethical Principles for Medical Research Involving Human Subjects. JAMA 2013, 310, 2191-2194. [CrossRef]

27. Johnstone, A.M.; Murison, S.D.; Duncan, J.S.; Rance, K.A.; Speakman, J.R. Factors influencing variation in basal metabolic rate include fat-free mass, fat mass, age, and circulating thyroxine but not sex, circulating leptin, or triiodothyronine. Am. J. Clin. Nutr. 2005, 82, 941-948. [CrossRef]

28. World Health Organization. Physical Status: The Use and Interpretation of Anthropometry. Report of a WHO Expert Committee; Technical Report Series no. 854; World Health Organization: Geneva, Switzerland, 1995.

29. Schofield, W.N. Predicting basal metabolic rate, new standards and review of previous work. Hum. Nutr. Clin. Nutr. 1985, 39 (Suppl. 1), 5-41.

30. Chowdhury, E.A.; Richardson, J.D.; Tsintzas, K.; Thompson, D.; Betts, J.A. Carbohydrate-rich breakfast attenuates glycaemic, insulinaemic and ghrelin response to ad libitum lunch relative to morning fasting in lean adults. Br. J. Nutr. 2015, 114, 98-107. [CrossRef]

31. Leidy, H.J.; Racki, E.M. The addition of a protein-rich breakfast and its effects on acute appetite control and food intake in 'breakfast-skipping' adolescents. Int. J. Obes. 2010, 34, 1125-1133. [CrossRef]

32. McCance, R.A.; Widdowson, E.M. McCance and Widdowson's the Composition of Foods, 7th ed.; Royal Society of Chemistry: London, UK, 2015.

33. Flint, A.; Raben, A.; Blundell, J.E.; Astrup, A. Reproducibility, power and validity of visual analogue scales in assessment of appetite sensations in single test meal studies. Int. J. Obes. 2000, 24, 38-48. [CrossRef]

34. Anderson, G.H.; Catherine, N.L.A.; Woodend, D.M.; Wolever, T.M.S. Inverse association between the effect of carbohydrates on blood glucose and subsequent short-term food intake in young men. Am. J. Clin. Nutr. 2002, 76, 1023-1030. [CrossRef]

35. Jansen, A.; Theunissen, N.; Slechten, K.; Nederkoorn, C.; Boon, B.; Mulkens, S.; Roefs, A. Overweight children overeat after exposure to food cues. Eat. Behav. 2003, 4, 197-209. [CrossRef]

36. Finlayson, G.; King, N.; Blundell, J. The role of implicit wanting in relation to explicit liking and wanting for food: Implications for appetite control. Appetite 2008, 50, 120-127. [CrossRef] [PubMed]

37. Oustric, P.; Thivel, D.; Dalton, M.; Beaulieu, K.; Gibbons, C.; Hopkins, M.; Blundell, J.; Finlayson, G. Measuring food preference and reward: Application and cross-cultural adaptation of the Leeds Food Preference Questionnaire in human experimental research. Food Qual. Prefer. 2020, 80, 103824. [CrossRef]

38. Griffioen-Roose, S.; Mars, M.; Siebelink, E.; Finlayson, G.; Tomé, D.; de Graaf, C. Protein status elicits compensatory changes in food intake and food preferences. Am. J. Clin. Nutr. 2011, 95, 32-38. [CrossRef] [PubMed]

39. Griffioen-Roose, S.; Mars, M.; Finlayson, G.; Blundell, J.E.; de Graaf, C. The effect of within-meal protein content and taste on subsequent food choice and satiety. Br. J. Nutr. 2011, 106, 779-788. [CrossRef] [PubMed]

40. Karl, J.P.; Cole, R.E.; Berryman, C.E.; Finlayson, G.; Radcliffe, P.N.; Kominsky, M.T.; Murphy, N.E.; Carbone, J.W.; Rood, J.C.; Young, A.J.; et al. Appetite Suppression and Altered Food Preferences Coincide with Changes in Appetite-Mediating Hormones During Energy Deficit at High Altitude, But Are Not Affected by Protein Intake. High Alt. Med. Biol. 2018, 19, 156-169. [CrossRef] [PubMed]

41. Patel, S.; Alvarez-Guaita, A.; Melvin, A.; Rimmington, D.; Dattilo, A.; Miedzybrodzka, E.L.; Cimino, I.; Maurin, A.-C.; Roberts, G.P.; Meek, C.L.; et al. GDF15 Provides an Endocrine Signal of Nutritional Stress in Mice and Humans. Cell Metab. 2019, 29, 707-718.e8. [CrossRef]

42. Cheke, L.G.; Bonnici, H.M.; Clayton, N.S.; Simons, J.S. Obesity and insulin resistance are associated with reduced activity in core memory regions of the brain. Neuropsychologia 2017, 96, 137-149. [CrossRef]

43. Matthews, D.R.; Hosker, J.P.; Rudenski, A.S.; Naylor, B.A.; Treacher, D.F.; Turner, R.C. Homeostasis model assessment: Insulin resistance and $\beta$-cell function from fasting plasma glucose and insulin concentrations in man. Diabetologia 1985, 28, 412-419. [CrossRef] 
44. Giezenaar, C.; Trahair, L.G.; Rigda, R.; Hutchison, A.T.; Feinle-Bisset, C.; Luscombe-Marsh, N.D.; Hausken, T.; Jones, K.L.; Horowitz, M.; Chapman, I.; et al. Lesser suppression of energy intake by orally ingested whey protein in healthy older men compared with young controls. Am. J. Physiol. Integr. Comp. Physiol. 2015, 309, R845-R854. [CrossRef]

45. Brindal, E.; Baird, D.; Danthiir, V.; Wilson, C.; Bowen, J.; Slater, A.; Noakes, M. Ingesting breakfast meals of different glycaemic load does not alter cognition and satiety in children. Eur. J. Clin. Nutr. 2012, 66, 1166-1171. [CrossRef] [PubMed]

46. Chandarana, K.; Drew, M.E.; Emmanuel, J.; Karra, E.; Gelegen, C.; Chan, P.; Cron, N.J.; Batterham, R.L. Subject Standardization, Acclimatization, and Sample Processing Affect Gut Hormone Levels and Appetite in Humans. Gastroenterology 2009, 136, 2115-2126. [CrossRef] [PubMed]

47. Trichopoulou, A.; Naska, A.; Costacou, T. DAFNE III Group Disparities in food habits across Europe. Proc. Nutr. Soc. 2002, 61, 553-558. [CrossRef] [PubMed]

48. Yannakoulia, M.; Karayiannis, D.; Terzidou, M.; Kokkevi, A.; Sidossis, L.S. Nutrition-related habits of Greek adolescents. Eur. J. Clin. Nutr. 2004, 58, 580-586. [CrossRef] [PubMed]

49. Rumm-Kreuter, D. Comparison of the eating and cooking habits of northern Europe and the Mediterranean countries in the past, present and future. Int. J. Vitam. Nutr. Res. 2001, 71, 141-148. [CrossRef]

50. Zandstra, E.H.; de Graaf, C. Sensory perception and pleasantness of orange beverages from childhood to old age. Food Qual. Prefer. 1998, 9, 5-12. [CrossRef]

51. Wadhera, D.; Capaldi-Phillips, E.D. A review of visual cues associated with food on food acceptance and consumption. Eat. Behav. 2014, 15, 132-143. [CrossRef]

52. Flint, A.; Raben, A.; Astrup, A.; Holst, J.J. Glucagon-like peptide 1 promotes satiety and suppresses energy intake in humans. J. Clin. Investig. 1998, 101, 515-520. [CrossRef]

53. Adrian, T.E.; Ferri, G.-L.; Bacarese-Hamilton, A.J.; Fuessl, H.S.; Polak, J.M.; Bloom, S.R. Human distribution and release of a putative new gut hormone, peptide YY. Gastroenterology 1985, 89, 1070-1077. [CrossRef]

54. De Silva, A.; Salem, V.; Long, C.J.; Makwana, A.; Newbould, R.D.; Rabiner, E.A.; Ghatei, M.A.; Bloom, S.R.; Matthews, P.M.; Beaver, J.D.; et al. The Gut Hormones PYY3-36 and GLP-17-36 amide Reduce Food Intake and Modulate Brain Activity in Appetite Centers in Humans. Cell Metab. 2011, 14, 700-706. [CrossRef] [PubMed]

55. Holst, J.J.; Schwartz, T.W.; Lovgreen, N.A.; Pedersen, O.; Beck-Nielsen, H. Diurnal profile of pancreatic polypeptide, pancreatic glucagon, gut glucagon and insulin in human morbid obesity. Int. J. Obes. 1983, 7, 529-538. [PubMed]

56. Näslund, E.; Barkeling, B.; King, N.; Gutniak, M.; Blundell, J.E.; Holst, J.J.; Rössner, S.; Hellström, P.M. Energy intake and appetite are suppressed by glucagon-like peptide-1 (GLP-1) in obese men. Int. J. Obes. 1999, 23, 304-311. [CrossRef] [PubMed]

57. Bartolomé, M.A.; Borque, M.; Martinez-Sarmiento, J.; Aparicio, E.; Hernández, C.; Cabrerizo, L.; Fernández-Represa, J.A. Peptide YY Secretion in Morbidly Obese Patients Before and After Vertical Banded Gastroplasty. Obes. Surg. 2002, 12, 324-327. [CrossRef] [PubMed]

58. Batterham, R.L.; Cohen, M.A.; Ellis, S.M.; Le Roux, C.W.; Withers, D.J.; Frost, G.S.; Ghatei, M.A.; Bloom, S.R. Inhibition of food intake in obese subjects by peptide YY3-36. N. Engl. J. Med. 2003, 349, 941-948. [CrossRef] [PubMed]

59. Vilsbøll, T.; Krarup, T.; Sonne, J.; Madsbad, S.; Vølund, A.; Juul, A.G.; Holst, J.J. Incretin Secretion in Relation to Meal Size and Body Weight in Healthy Subjects and People with Type 1 and Type 2 Diabetes Mellitus. J. Clin. Endocrinol. Metab. 2003, 88, 2706-2713. [CrossRef]

60. Kim, B.J.; Carlson, O.D.; Jang, H.J.; Elahi, D.; Berry, C.; Egan, J.M. Peptide YY is secreted after oral glucose administration in a gender-specific manner. J. Clin. Endocrinol. Metab. 2005, 90, 6665-6671. [CrossRef]

61. Vazquez Roque, M.I.; Camilleri, M.; Stephens, D.A.; Jensen, M.D.; Burton, D.D.; Baxter, K.L.; Zinsmeister, A.R. Gastric Sensorimotor Functions and Hormone Profile in Normal Weight, Overweight, and Obese People. Gastroenterology 2006, 131, 1717-1724. [CrossRef]

62. Morley, J.E.; Silver, A.J. Anorexia in the elderly. Neurobiol. Aging 1988, 9, 9-16. [CrossRef]

63. Datz, F.L.; Christian, P.E.; Moore, J. Gender-related differences in gastric emptying. J. Nucl. Med. 1987, 28, 1204-1207. 
64. Giezenaar, C.; Trahair, L.G.; Luscombe-Marsh, N.D.; Hausken, T.; Standfield, S.; Jones, K.L.; Lange, K.; Horowitz, M.; Chapman, I.; Soenen, S. Effects of randomized whey-protein loads on energy intake, appetite, gastric emptying, and plasma gut-hormone concentrations in older men and women. Am. J. Clin. Nutr. 2017, 106, 865-877. [CrossRef] [PubMed]

65. Savage, A.P.; Adrian, T.E.; Carolan, G.; Chatterjee, V.K.; Bloom, S.R. Effects of peptide YY (PYY) on mouth to caecum intestinal transit time and on the rate of gastric emptying in healthy volunteers. Gut 1987, 28, 166-170. [CrossRef]

66. Brennan, I.M.; Feltrin, K.L.; Nair, N.S.; Hausken, T.; Little, T.J.; Gentilcore, D.; Wishart, J.M.; Jones, K.L.; Horowitz, M.; Feinle-Bisset, C. Effects of the phases of the menstrual cycle on gastric emptying, glycemia, plasma GLP-1 and insulin, and energy intake in healthy lean women. Am. J. Physiol. Gastrointest. Liver Physiol. 2009, 297, 602-610. [CrossRef]

67. Hirschberg, A.L. Sex hormones, appetite and eating behaviour in women. Maturitas 2012, 71, $248-256$. [CrossRef] [PubMed]

68. Campolier, M.; Thondre, S.P.; Clegg, M.; Shafat, A.; Mcintosh, A.; Lightowler, H. Changes in PYY and gastric emptying across the phases of the menstrual cycle and the influence of the ovarian hormones. Appetite 2016, 107, 106-115. [CrossRef] [PubMed]

69. Cummings, D.E.; Purnell, J.Q.; Frayo, R.S.; Schmidova, K.; Wisse, B.E.; Weigle, D.S. A preprandial rise in plasma ghrelin levels suggests a role in meal initiation in humans. Diabetes 2001, 50, 1714-1719. [CrossRef] [PubMed]

70. Beasley, J.M.; Ange, B.A.; Anderson, C.A.M.; Miller, E.R., III; Holbrook, J.T.; Appel, L.J. Characteristics Associated With Fasting Appetite Hormones (Obestatin, Ghrelin, and Leptin). Obesity 2009, 17, 349-354. [CrossRef]

71. Sturm, K.; MacIntosh, C.G.; Parker, B.A.; Wishart, J.; Horowitz, M.; Chapman, I.M. Appetite, food intake, and plasma concentrations of cholecystokinin, ghrelin, and other gastrointestinal hormones in undernourished older women and well-nourished young and older women. J. Clin. Endocrinol. Metab. 2003, 88, 3747-3755. [CrossRef]

72. Bertoli, S.; Magni, P.; Krogh, V.; Ruscica, M.; Dozio, E.; Testolin, G.; Battezzati, A. Is ghrelin a signal of decreased fat-free mass in elderly subjects? Eur. J. Endocrinol. 2006, 155, 321-330. [CrossRef]

73. Di Francesco, V.; Zamboni, M.; Zoico, E.; Mazzali, G.; Dioli, A.; Omizzolo, F.; Bissoli, L.; Fantin, F.; Rizzotti, P.; Solerte, S.B.; et al. Unbalanced serum leptin and ghrelin dynamics prolong postprandial satiety and inhibit hunger in healthy elderly: Another reason for the "anorexia of aging". Am. J. Clin. Nutr. 2006, 83, 1149-1152. [CrossRef]

74. Schneider, S.M.; Al-Jaouni, R.; Caruba, C.; Giudicelli, J.; Arab, K.; Suavet, F.; Ferrari, P.; Mothe-Satney, I.; Van Obberghen, E.; Hébuterne, X. Effects of age, malnutrition and refeeding on the expression and secretion of ghrelin. Clin. Nutr. 2008, 27, 724-731. [CrossRef] [PubMed]

75. Giezenaar, C.; Luscombe-Marsh, N.D.; Hutchison, A.T.; Standfield, S.; Feinle-Bisset, C.; Horowitz, M.; Chapman, I.; Soenen, S. Dose-dependent effects of randomized intraduodenal whey-protein loads on glucose, gut hormone, and amino acid concentrations in healthy older and younger men. Nutrients 2018, 10, 78. [CrossRef]

76. Rigamonti, A.E.; Pincelli, A.I.; Corrá, B.; Viarengo, R.; Bonomo, S.M.; Galimberti, D.; Scacchi, M.; Scarpini, E.; Cavagnini, F.; Müller, E.E. Plasma ghrelin concentrations in elderly subjects: Comparison with anorexic and obese patients. J. Endocrinol. 2002, 175, R1-R5. [CrossRef]

77. Di Francesco, V.; Fantin, F.; Residori, L.; Bissoli, L.; Micciolo, R.; Zivelonghi, A.; Zoico, E.; Omizzolo, F.; Bosello, O.; Zamboni, M. Effect of age on the dynamics of acylated ghrelin in fasting conditions and in response to a meal. J. Am. Geriatr. Soc. 2008, 56, 1369-1370. [CrossRef] [PubMed]

78. Bauer, J.M.; Haack, A.; Winning, K.; Wirth, R.; Fischer, B.; Uter, W.; Erdmann, J.; Schusdziarra, V.; Sieber, C.C. Impaired postprandial response of active ghrelin and prolonged suppression of hunger sensation in the elderly. J. Gerontol. Ser. A Biol. Sci. Med. Sci. 2010, 65, 307-311. [CrossRef] [PubMed]

79. Nass, R.; Farhy, L.S.; Liu, J.; Pezzoli, S.S.; Johnson, M.L.; Gaylinn, B.D.; Thorner, M.O. Age-dependent decline in acyl-ghrelin concentrations and reduced association of acyl-ghrelin and growth hormone in healthy older adults. J. Clin. Endocrinol. Metab. 2014, 99, 602-608. [CrossRef]

80. Kojima, M.; Hosoda, H.; Date, Y.; Nakazato, M.; Matsuo, H.; Kangawa, K. Ghrelin is a growth-hormone-releasing acylated peptide from stomach. Nature 1999, 402, 656-660. [CrossRef] [PubMed] 
81. Wren, A.M.; Small, C.J.; Ward, H.L.; Murphy, K.G.; Dakin, C.L.; Taheri, S.; Kennedy, A.R.; Roberts, G.H.; Morgan, D.G.A.; Ghatei, M.A.; et al. The Novel Hypothalamic Peptide Ghrelin Stimulates Food Intake and Growth Hormone Secretion. Endocrinology 2000, 141, 4325-4328. [CrossRef] [PubMed]

82. Fernandez, G.; Cabral, A.; Cornejo, M.P.; De Francesco, P.N.; Garcia-Romero, G.; Reynaldo, M.; Perello, M. Des-Acyl Ghrelin Directly Targets the Arcuate Nucleus in a Ghrelin-Receptor Independent Manner and Impairs the Orexigenic Effect of Ghrelin. J. Neuroendocrinol. 2016, 28. [CrossRef]

83. Könner, A.C.; Klöckener, T.; Brüning, J.C. Control of energy homeostasis by insulin and leptin: Targeting the arcuate nucleus and beyond. Physiol. Behav. 2009, 97, 632-638. [CrossRef]

84. Serra-Prat, M.; Palomera, E.; Clave, P.; Puig-Domingo, M. Effect of age and frailty on ghrelin and cholecystokinin responses to a meal test. Am. J. Clin. Nutr. 2009, 89, 1410-1417. [CrossRef]

85. Doucet, E.; St-Pierre, S.; Alméras, N.; Mauriège, P.; Després, J.-P.; Richard, D.; Bouchard, C.; Tremblay, A. Fasting Insulin Levels Influence Plasma Leptin Levels Independently from the Contribution of Adiposity: Evidence from Both a Cross-Sectional and an Intervention Study1. J. Clin. Endocrinol. Metab. 2000, 85, 4231-4237. [CrossRef] [PubMed]

86. Saad, M.F.; Bernaba, B.; Hwu, C.-M.; Jinagouda, S.; Fahmi, S.; Kogosov, E.; Boyadjian, R. Insulin Regulates Plasma Ghrelin Concentration. J. Clin. Endocrinol. Metab. 2002, 87, 3997-4000. [CrossRef] [PubMed]

87. Saad, M.F.; Khan, A.; Sharma, A.; Michael, R.; Riad-Gabriel, M.G.; Boyadjian, R.; Jinagouda, S.D.; Steil, G.M.; Kamdar, V. Physiological insulinemia acutely modulates plasma leptin. Diabetes 1998, 47, 544-549. [CrossRef]

88. Ellis, A.C.; Chandler-Laney, P.; Casazza, K.; Goree, L.L.; McGwin, G.; Gower, B.A. Circulating ghrelin and GLP-1 are not affected by habitual diet. Regul. Pept. 2012, 176, 1-5. [CrossRef] [PubMed]

89. Erdmann, J.; Lippl, F.; Schusdziarra, V. Differential effect of protein and fat on plasma ghrelin levels in man. Regul. Pept. 2003, 116, 101-107. [CrossRef]

90. Veedfald, S.; Wu, T.; Bound, M.; Grivell, J.; Hartmann, B.; Rehfeld, J.F.; Deacon, C.F.; Horowitz, M.; Holst, J.J.; Rayner, C.K. Hyperosmolar Duodenal Saline Infusion Lowers Circulating Ghrelin and Stimulates Intestinal Hormone Release in Young Men. J. Clin. Endocrinol. Metab. 2018, 103, 4409-4418. [CrossRef] [PubMed]

91. Blom, W.A.M.; Lluch, A.; Stafleu, A.; Vinoy, S.; Holst, J.J.; Schaafsma, G.; Hendriks, H.F.J. Effect of a high-protein breakfast on the postprandial ghrelin response. Am. J. Clin. Nutr. 2006, 83, 211-220. [CrossRef]

Publisher's Note: MDPI stays neutral with regard to jurisdictional claims in published maps and institutional affiliations.

(C) 2020 by the authors. Licensee MDPI, Basel, Switzerland. This article is an open access article distributed under the terms and conditions of the Creative Commons Attribution (CC BY) license (http://creativecommons.org/licenses/by/4.0/). 